Revue d'histoire de l'Amérique française

RAS REVUE D.HISTOIRE DE L'AMÉRIQUE FRANÇAISE

\title{
Industries et politiques minières au Québec, une analyse économique 1896-1975
}

\section{Pierre Paquette}

Volume 37, numéro 4, mars 1984

URI : https://id.erudit.org/iderudit/304208ar

DOI : https://doi.org/10.7202/304208ar

Aller au sommaire du numéro

Éditeur(s)

Institut d'histoire de l'Amérique française

ISSN

0035-2357 (imprimé)

1492-1383 (numérique)

Découvrir la revue

Citer cet article

Paquette, P. (1984). Industries et politiques minières au Québec, une analyse économique 1896-1975. Revue d'histoire de l'Amérique française, 37(4), 573-602. https://doi.org/10.7202/304208ar d'utilisation que vous pouvez consulter en ligne.

https://apropos.erudit.org/fr/usagers/politique-dutilisation/ 


\title{
INDUSTRIES ET POLITIQUES MINIËRES AU QUÉBEC UNE ANALYSE ÉCONOMIQUE 1896-1975*
}

\author{
PIERRE PAQUETTE \\ Professeur-adjoint \\ Collège militaire royal de Saint-Jean
}

L'histoire économique générale de l'industrie minière au Québec est un domaine qui est relativement peu connu. Certes, il existe une historiographie de certains de ses principaux secteurs, celui de l'amiante en particulier, mais très peu d'études se sont donné une vocation analytique $^{1}$. L'importance de la demande internationale et le rôle du capital étranger dans l'essor définitif des régions minières au $\mathrm{XX}^{\mathrm{e}}$ siècle, ont, à juste titre, été soulignés, mais par contre, les conséquences économiques générales qui découlent de la structure de développement que ces facteurs ont engendrée n'ont pas été suffisamment examinées. C'est ce que nous nous proposons de faire dans les deux premières parties de cet article. La première partie examine la nature des importantes transformations des industries minières entre le $\mathrm{XIX}^{\mathrm{e}}$ et le $\mathrm{XX}^{\mathrm{e}}$ siècle tandis que la deuxième dégage certaines de leurs conséquences économiques. Si l'histoire générale du secteur minier est peu connue, celle de la politique minière l'est encore moins. Quelle est son origine? Quelle fut son évolution? Quels furent ses effets? Ce sont là les questions auxquelles nous nous attardons dans une troisième et dernière partie; nous chercherons tout particulièrement à relier la politique minière à la structure de développement de l'industrie.

\section{L'expansion et les mutations structurelles de l'industrie minière du $X I X^{e}$ au $X X^{e}$ siècle}

Au début du XIX ${ }^{\mathrm{e}}$ siècle, l'industrie minière québécoise se limitait essentiellement aux activités de deux secteurs, soit l'extraction de sable ferrugineux, en vue de la production de fonte dans des installations de

\footnotetext{
* Ce texte résume la thèse de doctorat de l'auteur, présentée et soutenue, avec succès, à l'Université McGill en 1982. Nous remercions le professeur Marc Vallières du département d'histoire de l'Université Laval, de même que les arbitres de cette revue, pour leurs commentaires et suggestions; plusieurs ont été incorporés au texte final. Nous demeurons, cependant, les seuls responsables des erreurs qui pourraient y être relevées.

L'historiographie de l'industrie de l'amiante, la plus volumineuse, est essentiellement descriptive; il en va de même avec les historiographies de la Beauce, de l'Abitibi-Témiscamingue et de la Côte nord; plusieurs de ces brochures ne fournissent que de l'information géologique. Certaines études sur l'amiante, cependant, n'ont pas ce caractère; voir par exemple les travaux de M.N. Mendells, The Asbestos Industry of Canada (Université McGill, Economic Studies, \#14, Montréal, 1930) et ceux de R. Armstrong, The Asbestos Industry in Quebec, 1878-1929, thèse de doctorat (Université Laval, 1978). Une importante source bibliographique de l'industrie minière est la liste annotée des publications du ministère de l'Énergie et des Ressources à Québec.
} 
type catalanes, et celles des carrières et sablières, reliées à l'industrie de la construction ${ }^{2}$. Les activités de ces industries étaient en général situées dans des régions déjà colonisées, donc près des sources de maind'oeuvre, et visaient tant bien que mal à combler une demande essentiellement locale; les forges érigées à Saint-Maurice sont les mieux connues de ces premières installations.

Après 1850 quatre nouveaux secteurs de l'industrie minière firent leur apparition, soit l'amiante, le cuivre, le phosphate et l'or. L'expansion des trois premiers fut provoquée non pas par l'expansion d'une demande locale, mais plutôt, et ce fut une nouvelle caractéristique de l'industrie, par une demande qui émanait de l'économie américaine ${ }^{3}$. Quant au secteur aurifère, son essor, après 1850 , fut le produit d'une ruée minière de nature spéculative, la première que connut le Québec, mais qui fut cependant d'une assez courte durée $e^{4}$.

Sur l'ensemble du XIX ${ }^{\mathrm{e}}$ siècle, les entreprises du secteur minier, organisées surtout par le capital anglo-canadien, puis britannique et américain, furent de tailles relativement restreintes ${ }^{5}$. Les techniques d'exploration minière demeuraient très artisanales; elles se limitaient à rechercher les effleurements de surface, le plus souvent dans des régions où des découvertes accidentelles avaient été rapportées et des anomalies signalées. Les techniques d'extraction, quant à elles, étaient plus élaborées mais demeuraient rudimentaires par comparaison à celles qui allaient être en usage au $\mathrm{XX}^{\mathrm{e}}$ siècle. Les techniques utilisées étaient surtout intensives en main-d'oeuvre, et de nature sélective, c'est-à-dire

2 Fauteux et Tessier ont bien décrit certaines de ces activités: voir J.-N. Fauteux, Essai sur l'industrie du Canada sous le régime français (Québec, 1927), chap. 1; A. Tessier, Les Forges de Saint-Maurice, 1729-1883 (Trois-Rivières, 1952).

3 L'exploitation du cuivre débuta en 1859 dans les Cantons de l'est et fut provoquée par les besoins de la guerre civile américaine; les premières expéditions d'amiante furent effectuées en 1878 tandis que l'extraction du phosphate date de 1875. Sources: Rapports du commissaire des terres de la Couronne (Québec, années diverses); J. Hamelin et Y. Roby Histoire économique et sociale du Québec, 1851-1896 (Montréal, 1971), 254-55 et Rapport sur les opérations minières de la province de Québec (Québec, années diverses). Certaines données chronologiques sur l'industrie cuprifère de l'époque sont regroupées dans Report on the Copper Deposits of the eastern townships of the Province of Québec (Dép. Colonisation, Mines et Pêcheries, Québec, 1915); sur l'industrie du phosphate, voir la brochure de H.C.C. Millar, intitulé Florida, South Carolina and Canadian Phosphates (Londres, 1891); sur l'amiante, voir en particulier Armstrong, op. cit., chap. $1-2$.

4 L'exploitation de l'or débuta après 1846 et fut déclenchée, comme ce fut souvent le cas dans l'industrie minière, par la découverte accidentelle d'une pépite dans la région de la Beauce. La plus grande partie de la soixantaine d'entreprises minières incorporées dans la province du Canada-Uni entre 1840 et 1867 , le furent en vue de travailler les dépôts alluviaux de la Beauce. Sources: W. Chapman, Mines d'or de la Beauce (Lévis, 1884); Statuts de la province du Canada (Québec, années diverses); T.A. Rickard, Man and Metals (New York, 1932), 628-629 et Notes on the Gold of Eastern Canada: being a reprint of portions of various reports of the geological survey of Canada from 1848 to 1863 (Montréal, 1864).

Par exemple, la douzaine d'entreprises actives dans le secteur de l'or vers 1890 utilisaient environ 300 mineurs, soit 25 par entreprise en moyenne; la douzaine d'entreprises actives dans le secteur du phosphate employaient chacune entre 30 et 110 travailleurs. Sources: Hamelin et Roby, idem. et Rapport du commissaire des terres de la Couronne, 1885-1895. 
qu'elles reposaient essentiellement sur les capacités des mineurs à repérer visuellement les filons d'apparence les plus riches; il en résultait évidemment une récupération très incomplète du minerai ${ }^{6}$. Peu de capital financier était requis en vue d'organiser l'exploitation; les nouvelles entreprises du secteur pouvaient donc être mises sur pied facilement et entrer rapidement en production. Une proportion très faible de ces entreprises était intégrée verticalement aux entreprises manufacturières locales, ou étrangères, à qui elles fournissaient la matière première; c'était là un facteur d'instabilité financière. Une exception existait à ce chapitre, soit l'industrie du minerai de fer qui était intégrée presque totalement aux fonderies localisées près des sources de minerai ${ }^{7}$.

Les caractéristiques que nous venons d'énumérer signifient que l'accès des entrepreneurs aux activités minières était relativement libre. L'industrie s'approchait donc du modèle de concurrence pure et parfaite que l'on retrouve dans les manuels de microéconomie, ce qui n'allait pas sans rendre leur existence précaire. Des six principaux secteurs de l'industrie minière au XIX ${ }^{\mathrm{e}}$ siècle, quatre (l'or, le cuivre, le phosphate et le minerai de fer) avaient presque complètement disparus au tournant du siècle; seul le secteur des matériaux de construction et celui de l'amiante demeuraient en expansion. Le déclin et la disparition éventuelle des secteurs précités furent imputables soit à la faiblesse des stocks et des teneurs par rapport à ceux de d'autres régions (ce fut la situation de l'or, du phosphate et du minerai de fer), soit à l'apparition de nouvelles technologies utilisées dans la transformation des minerais auxquelles l'économie québécoise ne put s'adapter. La production de minerai de fer, par exemple, chuta et disparut complètement au Québec après $1929^{8}$; la production de phosphate cessa, à toutes fins pratiques, lorsque furent mis en exploitation, vers la fin du siècle, les immenses dépôts de phosphate naturel de la Georgie et de la Floride ${ }^{9}$; quant aux

\footnotetext{
${ }^{6} \quad$ Les seules facettes de l'exploitation à être mécanisées le furent seulement vers la fin du $\mathrm{XIX}^{\mathrm{e}}$ siècle; elles se limitaient à certains services connexes, tels le pompage et la ventilation dans les mines souterraines, et le hissage, le transport et le forage dans les exploitations à ciel ouvert. C'est dans le secteur de l'amiante où la mécanisation apparut en premier au Québec. L'ensemble des nouvelles techniques provenaient de l'industrie minière américaine qui les avait adoptées après 1875. Sources: H. Barger et S.H. Schurr, The Mining Industries, 1899-1939, a Study of Output, Employment and Productivity (National Bureau of Economic Research, New York, 1944), 97106; Armstrong, op. cit., 57-58; le Rapport sur les opérations minières de la province de Québec fournit, de temps à autre, des descriptions des techniques de l'époque.

Une dizaine d'entreprises furent organisées entre 1856 et 1885 en vue de fondre les sables ferrugineux localisés à divers endroits du Québec; voir Tessier, op. cit., 151-53 et A. Faucher, Québec en Amérique au XIXe siècle (Fides, Montréal, 1973), 171-73.

8 Les raisons de cette disparition et de l'échec éventuel de toutes les entreprises du secteur eurent trait d'abord à l'incapacité de fondre adéquatement le fer titané et le fer sulfureux, puis à l'obsolescence de la technologie métallurgique de type catalane qui ne put survivre aux nouveaux procédés, le convertisseur Bessemer par exemple, qui permettaient de réaliser d'importantes économies d'échelle. Sources: Rapport sur les opérations minières de la province de Québec, 1897 1935, et Faucher, op. cit., 167-168.

${ }^{2}$ Rapport du commissaire des terres de la Couronne, 1885-1897 et Rapport sur les opérations minières de la province de Québec, 1897-1900.
} 
entreprises du secteur du minerai de cuivre, localisées dans les Cantons de l'est, elles disparurent une à une après la Première Guerre mondiale, étant les victimes d'un processus de substitution d'intrants utilisés dans la production d'acide sulfurique ${ }^{10}$.

La situation globale de l'industrie minière fut modifiée de façon remarquable lors des premières décennies du $\mathrm{XX}^{\mathrm{e}}$ siècle. Si les mutations technologiques provoquaient le déclin de certaines régions minières, elles contribuaient aussi à en créer de nouvelles. Peu élevée au XIX ${ }^{\mathrm{e}}$ siècle, la production minière québécoise s'accrut considérablement au début du $\mathrm{XX}^{\mathrm{e}}$, de sorte que nous pouvons affirmer que ce fut à cette époque que se constitua l'industrie minière contemporaine. Le tableau 1 résume l'évolution de la production de l'industrie. Entre 1900 et 1920, l'indice de la production minérale en dollars constants quintupla, et il quadrupla sur les deux décennies suivantes, soit au cours de la période 1920-1940. Le rythme général d'expansion de l'industrie, quoique plus faible, demeura néanmoins assez élevé après la Deuxième Guerre mondiale; entre 1940 et 1960 par exemple, la production en dollars constants tripla, ce qui équivaut à une hausse de $15 \%$ par année en moyenne.

Deux facteurs complémentaires expliquent l'essor marqué de l'industrie minière québécoise pendant la première moitié $\mathrm{du} \mathrm{XX}^{\mathrm{e}}$ siècle: le changement technologique et l'apparition de déséquilibres géographiques dans la répartition de l'offre mondiale.

Signalons d'abord que les nouvelles conjonctures du secteur minier québécois résultaient de l'accélération de l'industrialisation nord-américaine. Ce phénomène, qui débuta au tout début du siècle, fut qualifié de «nouvel industrialisme», et il constitua la deuxième phase de la révolution industrielle nord-américaine ${ }^{11}$. Ce «nouvel industrialisme» consacra l'alliance du progrès de la science avec les progrès techniques, et annonça l'arrivée d'un système industriel fondé sur les productions lourdes et caractérisé par la fabrication sur de grandes échelles. La métallurgie, en particulier, devint l'un des secteurs stratégiques de la nouvelle croissance économique ${ }^{12}$; l'amélioration des connaissances

\footnotetext{
10 En 1919, est organisée, aux États-Unis, la Texas Gulf Sulphur Co. qui fut responsable du remplacement de la pyrite de cuivre, le minerai des Cantons de l'est, par le soufre brut dans la fabrication d'acide sulfurique. À l'époque, le minerai québécois, de faible teneur, n'était utilisé qu'à cette fin. L'innovation de la Texas Gulf fit chuter les cours de l'acide sulfurique; Rapport sur les opérations minières de la province de Québec, 1916-1927.

11 L'expression «nouvel industrialisme» est de W.H. Aitken et W.T. Easterbrook, dans Canadian Economic History (Toronto, 1956), 517. La première phase de la révolution industrielle fut amorcée, sur le plan technique, par la substitution du moulin à eau par l'engin à vapeur comme source principale de force motrice, et par le remplacement du charbon de bois par le charbon minéral comme source principale de chaleur industrielle; les températures de fonte furent élevées, ce qui améliora considérablement la qualité des produits; sur ces questions voir, par exemple, T.S. Aston, The Industrial Revolution, 1760-1830 (Londres, 1948), 46-48.

12 J.-P. Rioux, La Révolution industrielle, 1780-1880 (Paris, 1971), 81.
} 
TABLEAU 1

STATISTIQUES DE LA PRODUCTION MINIÈRE AU QUÉBEC, 1890-1975

\begin{tabular}{|c|c|c|c|}
\hline \multirow{2}{*}{ ANNÉES } & \multicolumn{2}{|c|}{ VALEUR DE LA PRODUCTION } & INDICE DE LA PRODUCTION \\
\hline & (millions \$) & (millions \$ constants) & (millions \$ constants) \\
\hline & & $1911=100$ & $1900=100$ \\
\hline 1890 & 1,2 & 1,1 & 41 \\
1900 & 2,6 & 2,7 & 100 \\
1911 & 8,7 & 8,7 & 320 \\
1920 & 28,3 & 13,6 & 501 \\
1930 & 41,2 & 25,1 & 928 \\
1940 & 87,7 & 48,1 & 298 \\
1950 & 220,6 & 79,7 & 5151 \\
1960 & 446,6 & 139,1 & 6951 \\
1970 & 811,9 & 187,7 & 6551 \\
1975 & 1271,5 & 176,9 & 28 \\
\hline
\end{tabular}

Sources: Les données de la production minérale monétaire des années indiquées au tableau proviennent de: Rapport annuel, Dép. des terres de la Couronne (1890), Rapport sur les opérations minières de la province de Québec $(1930,1940)$ et Annuaire statistique du Québec (1950, 1960, 1970, 1975). La production minérale totale n'a été recencée rigoureusement qu'après 1897 alors que débuta la publication régulière du Rapport sur les opérations minières. L'indice des prix utilisé en vue de dériver la production en dollars constants est un indice pondéré des prix du fer et des produits du fer, des métaux non ferreux et des produits minéraux non métalliques; les coefficients de pondération utilisés correspondent à l'importance de chaque groupe de minerai dans la production minière totale. Les données sur les prix proviennent de: H. Michell, «Statistics of Prices», dans K. Taylor et H. Michell, Statistical Contributions to Canadian Economic History (Toronto, 1931), 2: 56, 82 et tableau A en annexe du volume, M.C. Urquhart et K.A.H. Buckley, Historical Statistics of Canada (Toronto 1965), 293 et Prix et indices de prix, Statistique Canada, \#62-002. Pour l'année 1890, les coefficients de pondération et les prix retenus furent ceux du cuivre (environ $55 \%$ de la production totale) et des minéraux non métalliques (environ $45 \%$ de la production totale); tel que suggéré précédemment les données antérieures à 1897 sont très approximatives. Dans nos calculs des coefficients de pondération, nous avons regroupé le groupe des matériaux de construction au groupe des minéraux non métalliques.

dans ce domaine rendit possible, entre autres, la récupération efficace de nouvelles substances minérales, telles que l'aluminium et les métaux non ferreux. Par ailleurs, la disponibilité et l'usage généralisé de ces nouveaux matériaux furent en grande partie la conséquence de l'apparition de deux nouvelles sources de forces motrices: l'électricité et le moteur à explosion. Ces nouvelles données technologiques, que nous résumons très brièvement ici, accélérèrent l'industrialisation du continent et provoquèrent d'importantes mutations structurelles. L'essor des industries lourdes fut stimulé, et de tous nouveaux secteurs industriels furent organisés, en particulier ceux reliés à la fabrication de matériel de transport et de produits électriques. Les nouvelles technologies contribuèrent aussi à intégrer davantage entre eux les procédés indus- 
triels. Ces intégrations, associées aux échelles de production de plus en plus élevées, donna lieu, le plus souvent par des fusions, à l'organisation de grandes entreprises nationales et multinationales ${ }^{13}$. Ces tendances globales furent perpétuées d'une décennie à l'autre et prirent un nouvel envol après la Seconde Guerre mondiale avec l'essor très rapide des industries de biens de consommation durable.

Les mutations que nous venons de décrire résultèrent en des hausses marquées de demandes de matières premières, dont évidemment les ressources minérales; la production des industries minières nord-américaines et mondiales connurent en conséquence des expansions considérables $^{14}$. Un problème majeur apparut cependant très rapidement au début du siècle: la demande provenant des principaux pays consommateurs vint à dépasser les capacités de production de plusieurs de leurs industries minières ${ }^{15}$. De nouvelles régions productrices devaient donc être trouvées, et développées, afin d'éliminer les déséquilibres qui s'étaient créés, ou qui allaient l'être, entre la répartition géographique des demandes et celle de la production. Les premières décennies du $\mathrm{XX}^{\mathrm{e}}$ siècle virent donc le début de l'internationalisation des industries minières; l'exploration et l'exploitation se transformèrent en activités d'envergure internationale. Le contrôle par les industries consommatrices des meilleures sources d'approvisionnement était devenu un facteur fondamental à leur survie d'autant plus que leurs besoins en minerais allaient être de plus en plus comblés par des importations ${ }^{16}$. Quoique plusieurs techniques de contrôle aient été disponibles, la plus utilisée fut la propriété directe via l'organisation de filiales étrangères et de consortiums internationaux. À cet égard, les États-Unis et l'Angleterre jouèrent un rôle de tout premier plan; les capitaux britanniques parvenaient rapidement à prendre contrôle des principaux gîtes de minerais ferreux et métalliques de l'Australie, de la Rhodésie, du Congo et de l'Afrique du sud; le contrôle du capital américain s'étendit aux gisements importants de presque tous les pays de l'Amérique latine, y compris la région des Caraïbes, de même que, conjointement avec le capital

13 Ashton, op. cit., 53

14 La production mondiale de minerai de fer augmenta de $73 \%$ entre 1925 et 1942 , celle du cuivre de $40 \%$ et celle de l'amiante, entre 1935-45, de $77 \%$. Entre 1945 et 1975 les hausses respectives furent de $186 \%$, $183 \%$ et $380 \%$. Sources: U.S. Minerals Yearbook (U.S. Bureau of Mines, Washington D.C., années diverses) et Rapport final, Commission d'enquête sur les perspectives économiques canadiennes (Ottawa, 1957), Annexe E.

15 Les premières décennies du siècle virent surgir aux États-Unis le spectre de l'épuisement éventuel des réserves de minerais de nickel, de cuivre, de platine, de fer, d'or et d'argent; des carences importantes se manifestaient aussi pour le mica, le manganèse et quelques autres minerais; en Angleterre, l'épuisement touchait les minerais de cuivre, de plomb, de fer et d'étain. C.K. Leith, World Minerals and World Politics (New York, 1931), 23-24.

i6 Après 1945 par exemple, les États-Unis devenaient un importateur net de presque tous les métaux de base. Cette situation inquiétante avait été analysée dans Ressources for Freedom, a report to the President by the President's Materials Policy commission (Washington, D.C., juin 1952). 
anglais, sur les puits pétroliers du Moyen-Orient ${ }^{17}$. Le résultat de tout cela fut une modification graduelle, mais significative, de la localisation de la production minière mondiale. Par exemple, les parts relatives dans la production mondiale de certains minerais du plus important consommateur, les États-Unis, baissèrent de façon marquée ${ }^{18}$, tandis que celles de nouvelles régions, la Zambie et le Chili (pour le cuivre) et le Vénézuela (pour le minerai de fer), évoluèrent en direction opposée $^{19}$.

L'expansion de la production minière québécoise et mondiale au $\mathrm{XX}^{\mathrm{e}}$ siècle fut le produit non seulement des hausses considérables des demandes de minerai, mais aussi des nouvelles technologies de l'exploitation et des diverses étapes de leur transformation primaire. Ces nouvelles technologies diminuèrent les coûts de production et provoquèrent des restructurations majeures des industries extractives. Avant de dégager les conséquences structurelles de ces transformations nous décrirons rapidement la nature et le sens de l'évolution technologique en cause.

Essentiellement sélective au XIXe siècle, la technologie de l'extraction minière devint non sélective au $\mathrm{XX}^{\mathrm{e}}$. Cela signifiait que l'exploitation d'un gîte allait être mécanisée; la discrimination visuelle du mineur quant aux filons à extraire était, en quelque sorte, remplacée par la voracité d'une pelle mécanique. Cette mécanisation de l'extraction n'était possible, par ailleurs, que parce qu'elle avait été précédée de modifications des techniques utilisées dans la récupération des substances métalliques à partir des minerais. L'amélioration de ces techniques, et les nouvelles qui furent mises au point ${ }^{20}$, permettaient la mécanisation de l'extraction parce que la séparation des minéraux du roc devenait davantage fondée sur les propriétés physiques et chimiques

\footnotetext{
17 La France, via son système colonial, exerça également un contrôle sur les gisements localisés en Afrique du nord et en Nouvelle Calédonie; Leith, op. cit., 55-57, 60-61. Il est important de noter aussi que l'amélioration des réseaux de transport, et leurs extensions, fut un facteur important qui accéléra l'intégration des diverses régions économiques du continent et du monde.

${ }_{18}$ Les parts américaines de la production mondiale de minerai de fer et de cuivre, par exemple, deux minéraux importants dans le «nouvel industrialisme», passèrent de $37 \%$ à $45 \%$ respectivement à $11 \%$ et $23 \%$ entre 1945 et 1975. Source: U.S. Minerals Yearbook, 1945 et 1975.

${ }_{19}$ En 1945, la Zambie, le Zaïre et le Chili comptaient pour $29 \%$ de la production mondiale de cuivre; en 1975, leurs parts totales avaient augmenté à 36\%. Idem.

20 Le procédé de cyanisation dans la récupération de l'or, le procédé Mond-Orford de séparation des minerais non ferreux et le procédé de «flottation» appliqué aux minerais mixtes cuivre-plomb-zinc furent tous mis au point au tournant du siècle; ils nécessitaient tous une alimentation en minerai finement broyé, d'où l'importance que prenait pour ces procédés, l'étape de la préparation du minerai après extraction. Sources: Barger et Schurr, op . cit., 151, 155, Easterbrook et Aitken, op. cit., 535 et H.A. Innis, «Settlement and the Mining Frontier», dans W.A. MacKintosh et W.L.G. Joerg, Canadian Frontiers of Settlement (Toronto, 1936), 9: 338. Un résumé de la transformation des technologies minières du $\mathrm{XIX}^{\mathrm{e}}$ au XX $\mathrm{XX}^{\mathrm{e}}$ siècles et de celles utilisées dans le secteur de l'amiante, du cuivre et minerai de fer se trouve dans Pierre Paquette, L'extraction de matières premières et la politique minière de l'État: une analyse de leur évolution et de leur contribution au développement économique du Québec; 1867-1975, thèse de doctorat nonpubliée (Université McGill, 1982), 168-189.
} 
des composés extraits du sol. Aussi, la plupart de ces techniques de transformation primaire exigeaient, afin d'assurer un recouvrement maximal, une alimentation en minerai préalablement concassé et finement broyé. Les moulins de concentration devenaient ainsi l'étape intermédiaire essentielle entre l'extraction et la récupération finale ${ }^{21}$. Ces équipements de transformation primaire furent continuellement perfectionnés et firent de plus en plus appel à une préparation soignée du minerai ${ }^{22}$. La hausse considérable dans leurs capacités, le produit d'économies d'échelle, se répercuta évidemment en amont, de sorte que les opérations proprement minières se transformèrent souvent en chantiers aux proportions gigantesques. Durant les années 1950, par exemple, le volume annuel extrait d'un gisement de minerai de fer dépassait rarement deux millions de tonnes; 15 ans plus tard, la capacité de certaines exploitations atteignait 20 millions de tonnes ${ }^{23}$.

L'augmentation considérable des échelles optimales de production fut l'une des conséquences les plus importantes des nouvelles technologies de l'extraction et de la transformation. C'est sans doute le facteur individuel qui explique le mieux les mutations structurelles de l'industrie minière nord-américaine et mondiale pendant la première moitié du $\mathrm{XX}^{\mathrm{e}}$ siècle. Si l'entreprise-type de l'industrie minière du $\mathrm{XIX}^{\mathrm{e}}$ siècle était peu capitalisée et de taille restreinte, son vis-à-vis du $\mathrm{XX}^{\mathrm{e}}$ siècle était tout le contraire: ses échelles de production devinrent très élevées et ses techniques hautement intensives en capital. De compétitives qu'elles étaient au XIX ${ }^{\mathrm{e}}$ siècle, les structures des secteurs miniers devenaient très concentrées. Seules les entreprises minières et manufacturières déjà établies possédaient l'entrepreneurship, l'expertise techni-

\footnotetext{
21 Barger et Schurr, op. cit., 146; soulignons ici que la mécanisation avancée de l'exploitation minière fut avant tout stimulée par la baisse générale des teneurs; en fait, c'est l'épuisement graduel des gîtes de teneur élevée qui amorça l'introduction de procédés de récupération qui rentabilisaient l'exploitation de gîtes de teneurs plus faibles. Barger et Schurr, op . cit., 108, 114.

22 Dans le secteur sidérurgique, par exemple, la baisse des teneurs des gîtes miniers mena à la concentration de minerais de faible teneur avant l'alimentation des hauts-fourneaux; ibid., 148. Plus tard, l'on constata qu'un minerai finement broyé puis enrichi diminuait la consommation de coke par les hauts fourneaux en donnant lieu à une oxydation plus rapide du minerai. Sources: G. Manners, The Changing World Market for Iron Ore, 1950-1980 (Baltimore et Londres, 1971), 36; une étude exhaustive de l'évolution technologique de l'industrie du minerai de fer et de la fabrication de l'acier se trouve dans W.T. Hogan, Economic History of the Iron and Steel Industry of the United States (5 volumes, Lexington, Toronto et Londres, 1971).

${ }_{23}$ Manners, op . cit., 165-166. La taille des équipements miniers augmenta de façon marquée au $\mathrm{XX}^{\mathrm{e}}$ siècle; la capacité des pelles mécaniques, utilisées dans les exploitations à ciel ouvert, passa de 1.5 verges cubes en 1914 à 8 verges cubes en 1950; celle des camions augmenta de 10 à 100 tonnes entre 1950 et 1965 ; d'importantes économies d'échelles étaient ainsi réalisées. L'usage de l'électricité fut par ailleurs un facteur d'ordre général qui contribua à faire baisser les coûts d'exploitation; l'historien Innis, par exemple, note que l'électrification fit diminuer de $50 \%$ les coûts de concentration de l'industrie minière du nord-est ontarien. Sources: G. Manners, op . cit., 159; A.R. Dennis, The Free World's Largest Asbestos Producer (Canadian Johns Manville Co. Ltd., mai 1967) et Innis, op. cit., 65. Pour une description de l'importance de la mécanisation dans le secteur de l'amiante, voir J.G. Ross, L'Amiante chrysotile au Canada (Ottawa, 1934), 41-42; pour une description des changements intervenus dans la taille des moulins de concentration au début du siècle dans le nord-est de l'Ontario, voir Innis, op. cit., 331, 359, 380-381.
} 
que et le capital financier que pouvait nécessiter l'organisation de nouvelles unités de production. C'est ainsi qu'apparut la structure oligopole de l'industrie minière; une fois constituée, elle eut tendance à s'accentuer car l'importance des frais fixes dans les nouvelles structures des coûts favorisait la monopolisation des gîtes, via les prises de contrôle et l'achat de droits aux minéraux, afin d'alimenter les équipements en place.

La section suivante examine la structure de développement du secteur minier québécois; nous nous penchons plus particulièrement sur certaines de ses conséquences majeures sur le développement d'ensemble de l'économie du Québec.

\section{La structure de développement de l'industrie minière et les conséquences de la satellisation}

La croissance de l'industrie minière québécoise se réalisa, ainsi que nous l'avons souligné plus haut, dans le contexte général des transformations économiques et technologiques que subit l'économie nordaméricaine vers la fin du XIX et au début du $\mathrm{XX}^{\mathrm{e}}$ siècle. Le Québec devint l'une des régions du monde qui fut appelée à combler les demandes de minerais des régions économiques plus industrialisées qui l'entouraient ${ }^{24}$.

Trois secteurs de l'industrie minière québécoise connurent une expansion importante au $\mathrm{XX}^{\mathrm{e}}$ siècle: l'amiante, le cuivre et le minerai de fer. L'évolution de la production de ces minerais durant la période 1900-1975 est présentée au tableau 2. L'amiante fut le premier secteur à connaître un essor important; en effet, le volume de production de ce minerai augmentait près de 16 fois entre 1900 et 1925 . Le secteur du cuivre fut le deuxième; l'expansion de ce secteur, qui se déroula après 1924, coïncidait avec l'organisation de la Noranda Mines Ltd. et l'ouverture d'une nouvelle région minière: le nord-ouest du Québec. Le troisième secteur, celui du minerai de fer, fut organisé après la Deuxième Guerre mondiale; ce secteur fut aussi associé à l'ouverture d'une nouvelle «frontière», soit la région du Nouveau-Québec. Ces trois secteurs

\footnotetext{
24 Durant la période 1945 à 1975, par exemple, la production québécoise d'amiante, de cuivre et de minerai de fer compta en moyenne pour $50 \%, 2,5 \%$ et $2 \%$ respectivement de la production mondiale; la production du Québec dépassa donc de beaucoup la consommation locale. Ces pourcentages ont été calculés d'après les données de U.S. Minerals Yearbook, et de L'Industrie minière de la province de Québec.
} 
furent, et de loin, les plus importants de l'industrie minière québécoise sur l'ensemble du XX' $\mathrm{XX}^{\mathrm{e}}$ siècle $^{25}$.

TABLEAU 2

ÉVOLUTION DE LA PRODUCTION ET DES EXPORTATIONS D'AMIANTE, DE CUIVRE ET DE MINERAI DE FER, QUÉBEC, 1900-1975

\begin{tabular}{|c|c|c|c|c|c|}
\hline \multirow[t]{2}{*}{ MINERAI } & \multicolumn{5}{|c|}{ ANNÉES } \\
\hline & 1900 & 1925 & 1945 & 1965 & 1975 \\
\hline \multicolumn{6}{|l|}{ Amiante } \\
\hline $\begin{array}{l}\text { production } \\
\text { (milliers tonnes) }\end{array}$ & 18,2 & 283,5 & 466,9 & 1234,9 & 1014,8 \\
\hline $\begin{array}{l}\text { - exportations } \\
\text { (\% production) }\end{array}$ & N.D. & 91 & 94 & 95 & 100 \\
\hline \multicolumn{6}{|l|}{ Cuivre } \\
\hline $\begin{array}{l}\text { - production* } \\
\text { (milliers tonnes) }\end{array}$ & 1,1 & 1,3 & 51,3 & 173,6 & 129,7 \\
\hline $\begin{array}{l}\text { - exportations } \\
\text { (\% production) }\end{array}$ & N.D. & 92 & $55^{*}$ & $17 *$ & $43^{3}$ \\
\hline \multicolumn{6}{|l|}{ Minerai de fer } \\
\hline $\begin{array}{l}\text { - production } \\
\text { (milliers tonnes) }\end{array}$ & 0,1 & NIL & NIL & 14,8 & 12,7 \\
\hline $\begin{array}{l}\text { - exportations } \\
\text { (\% production) }\end{array}$ & N.D. & - & - & 100 & 100 \\
\hline \multicolumn{6}{|c|}{$\begin{array}{l}\text { *: indique le contenu métallique des minerais; le pourcentage de minerai de cuivre exporté } \\
\text { réfère à la production canadienne; les données pour } 1945,1965 \text { et } 1975 \text { ne sont pas repré- } \\
\text { sentatives de la situation québécoise car la quasi-totalité du cuivre brut extrait fut raffinée } \\
\text { au Québec. } \\
\text { N.D.: non disponible } \\
\text { Sources: Report on the Mining and Metallurgical Industries of Canada, Annual Report on the } \\
\text { Mineral Production of Canada, Canadian Minerals Yearbook, Gouvernement du Canada, et } \\
\text { Canadian Mining Journal. }\end{array}$} \\
\hline
\end{tabular}

La structure de développement de ces trois principaux secteurs de l'industrie fut essentiellement de type satellitique: tous les principaux facteurs ayant favorisé leur organisation et leur expansion, à l'exception d'un seul (soit la politique minière), provenaient de centres économiques localisés à l'extérieur du Québec. La technologie de l'extraction et les nouvelles demandes, par exemple, provenaient de centres éco-

\footnotetext{
25 Durant la première moitié du siècle, l'amiante et le cuivre seuls comptaient pour environ $40 \%$ de la production minérale totale du Québec; après 1954, suite à l'expansion de l'exploitation du minerai de fer, les trois minerais ensemble comptaient pour $60 \%$ du total. Sources des données: les mêmes qu'au tableau 2. Il existe d'autres secteurs importants de l'industrie minière québécoise: le zinc, l'or et le groupe des matériaux de construction, par exemple. Les trois secteurs retenus sont les plus importants; ils décrivent bien les tendances globales de l'industrie minière. C'est leur développement aussi qui fit naître d'importants espoirs d'industrialisation; c'est cet aspect qui est examiné dans cette section.
} 
nomiques externes, surtout des États-Unis. Ainsi que le révèle le tableau 2 , plus de $90 \%$ de la production d'amiante et de cuivre était exporté à l'état brut au début du siècle; une prépondérance analogue des exportations dans la production totale caractérisa aussi l'industrie du minerai de fer après 1950. Le Québec devenait l'un des plus importants fournisseurs de l'économie des États-Unis; de 1945 à 1975, les importations d'amiante, de cuivre et de minerai de fer en provenance du Québec comptaient respectivement pour $90 \%, 20 \%$ et $15 \%$ des importations américaines totales de ces produits ${ }^{26}$.

La satellisation de l'industrie minière québécoise fut accentuée de façon importante par la structure de la propriété de l'industrie. En effet, l'organisation des trois principaux secteurs de l'industrie fut effectuée essentiellement par le capital anglo-canadien et par l'expansion du capitalisme américain outre-frontière. L'industrie de l'amiante et, plus tard, celle du minerai de fer furent organisées et prises en main très tôt par le capital américain ${ }^{27}$. Ce déplacement du capital américain au Québec se réalisa dans le cadre de stratégies internationales d'intégration verticale et horizontale développées par les grandes corporations manufacturières qui s'étaient constituées dans le secteur sidérurgique et de l'industrie de la fabrication des produits d'amiante ${ }^{28}$. Ces stratégies visaient ni plus ni moins qu'à transformer en dépôts captifs les divers gîtes miniers qui pourraient être localisés à l'extérieur des frontières des EtatsUnis. L'objectif ultime des nouveaux ensembles oligopolistiques était l'acquisition d'un plus grand pouvoir de marché par la création de bar-

26 Le marché américain était de loin la destination principale des exportations québécoises de minerais; les données ont été dérivées à partir de renseignements fournis dans le U.S.Minerals Yearbook et Canadian Minerals Yearbook, années diverses. Les pourcentages furent calculés en multipliant la part canadienne des importations américaines par la part québécoise de la production canadienne de chaque produit.

27 La propriété américaine de l'industrie de l'amiante date du début du siècle; elle fut réalisée principalement par des prises de contrôle de la part de manufacturiers de produits d'amiante localisés aux États-Unis. La propriété américaine du secteur du minerai de fer date de l'organisation même de l'industrie après la guerre; les droits miniers dans les zones minéralogiques les plus favorables furent rapidement monopolisés par les principales sidérurgies américaines et ontariennes. Une bonne chronologie des prises de contrôle dans l'industrie de l'amiante entre 1878 et 1929 se trouve dans Armstrong, op. cit.; pour l'histoire de l'industrie entre 1925 et 1975, voir Paquette, op. cit., 213-223. Le développement de l'industrie du minerai de fer au NouveauQuébec est examiné de façon détaillée dans ibid., 269-303.

28 Une structure mondiale oligopolistique, dominée par des entreprises américaines et anglaises manufacturières de produits d'amiante, fut établie dans le secteur de l'extraction vers la fin du XIX ${ }^{\mathrm{e}}$ siècle; les décennies subséquentes furent caractérisées par des prises de contrôle d'entreprises minières indépendantes. En 1901, par exemple, la H.W. John's Manville prenait le contrôle au Québec de la Asbestos and Asbestic Co.; en 1906, la Keasby et Mattison Co., une autre entreprise manufacturière américaine, prenait le contrôle de la Bell Asbestos Co. L'oligopole du fer et de l'acier apparut au début du $\mathrm{XX}^{\mathrm{e}}$ siècle, du moins aux États-Unis, à la suite de fusions d'entreprises qui étaient déjà, à l'époque, intégrées à l'étape de l'extraction des matières premières. Sources: Armstrong, op. cit., 32, 140-41, Manners, op. cit., 320, Hogan, op . cit., 95-97, et R.L. Nelson, Merger Movements in American Industry, 1895-1956 (National Bureau of Economic Research, Princeton, 1959), 154-55, 161. 
rières d'entrée aux industries en cause ${ }^{29}$. C'est une stratégie qui réussit pleinement, mais l'un de ses effets fut la subordination d'industries minières nationales à des industries manufacturières étrangères; c'est cette caractéristique qui domina la structure de développement de l'industrie québécoise de l'amiante et du minerai de $\mathrm{fer}^{30}$. Le tableau 3 rappelle certaines des principales caractéristiques des entreprises de ces deux secteurs au Québec. Les renseignements présentés indiquent que six des sept entreprises du secteur de l'amiante étaient, au milieu de la dernière décennie, intégrées à des entreprises étrangères, américaines pour la plupart, manufacturières de produits d'amiante; la septième, l'Asbestos Corporation, était la seule entreprise indépendante du secteur. Ce portrait d'ensemble rejoint globalement celui rapporté pour le secteur du minerai de fer.

L'organisation et l'évolution de l'industrie du cuivre et l'industrie des minerais non ferreux en général diffèrent sensiblement de celles de ces deux secteurs. Si l'amiante et le minerai de fer furent organisés par le capital et l'entrepreneurship américain, le secteur cuprifère, lui, fut développé presqu'exclusivement par le capital et l'entrepreneurship anglo-canadien. En effet, l'organisation de ce secteur résulta du débordement au Québec d'un spectaculaire boom minier dans le nord-est ontarien. Cet essor, qui prit, au début du siècle, les proportions d'un véritable «El dorado», fut à l'origine d'une importante accumulation de capital, et de l'établissement de Toronto en tant que premier centre financier de l'industrie minière de propriété canadienne ${ }^{31}$. Quoique l'expansion du secteur cuprifère au Québec ait été aussi reliée à l'expansion de la demande américaine de minerais, sa structure de développement fut beaucoup moins dépendante, ou satellitique, que celle de l'amiante et du minerai de fer. Non seulement le secteur de l'extraction fut-il tenu à l'écart de toute intégration corporative à des complexes manufacturiers américains, mais en plus, la richesse des gisements

29 Le Québec fut seulement l'un des nouveaux sites d'activités choisis; dans le cas du minerai de fer, par exemple, les sidérurgies américaines furent aussi actives au Chili, au Brésil, au Vénézuela et au Libéria en Afrique. Manners, op. cit., 229, Hogan, op. cit., 1481-82, 1659.

30 Les études empiriques sur le comportement d'entreprises oligopolistiques et sur la question de barrières d'entrée abondent; voir, par exemple, J.S. Bain, Barriers to New Competition (Cambridge, 1956) et F.M. Scherer, Industrial Market Structure and Economic Performance (Chicago, 1970); certaines études statistiques, résumées dans Scherer, op. cit., 231, indiquent qu'il existe une corrélation positive entre les taux de profit d'industries et les difficultés, ou barrières, qui bloquent l'organisation d'entreprises rivales; l'absence de contrôle des sources de matières premières constitue l'une des difficultés qu'une entreprise doit surmonter en vue de devenir et de demeurer rentable.

31 L'histoire minière de la région débuta en 1902 lorsque fut commencée la construction, le long de la frontière Québec - Ontario, de la Timiskaming and Northern Ontario Railway de North Bay jusqu'à Cokrane, environ $200 \mathrm{~km}$ plus au nord. Ce projet donna lieu à d'importantes découvertes minières: dépôts aurifères de Porcupine Lake (1909) et de Kirkland Lake (1911) et de cuivre et de zinc à Timmins (1920); ce fut l'origine des empires miniers Dome, Hollinger et McIntyre; sources: E.G. Pye, One Hundred Years of Mining in Ontario (Dept of Mines, Toronto, 1967), 8, 10-12; pour un récit détaillé des événements associés à ces entreprises voir Innis, op. cit., 349-371 
TABLEAU 3

PRINCIPALES CARACTÉRISTIQUES DES PRODUCTEURS D'AMIANTE ET DE MINERAI DE FER AU QUÉBEC (1975)

\begin{tabular}{|c|c|c|}
\hline Entreprise & $\begin{array}{l}\% \text { de la } \\
\text { production } \\
\text { totale du } \\
\text { secteur** }\end{array}$ & Liens de propriété $* * *$ \\
\hline $\begin{array}{l}\text { Amiante } \\
\text { Asbestos Corp. (1879)* } \\
\text { Canadian Johns Manville (1882) } \\
\text { Flint Kote Mines Ltd. (1946) } \\
\text { Bell Asbestos Mines (1888) } \\
\text { Carey Canadian Mines (1958) } \\
\text { Lake Asbestos of Québec (1958) } \\
\text { National Asbestos Mines Ltd (1958) }\end{array}$ & $\begin{array}{r}30 \\
39 \\
3 \\
4 \\
7 \\
11 \\
6\end{array}$ & $\begin{array}{l}\text { General Dynamics }^{\mathrm{a}} \\
\text { Johns Manville Corp. } \\
\text { Flint Kote Corp. } \\
\text { Turner and Newall Ltd. } \\
\text { Philip Carey Mfg. Co. } \\
\text { American Smelting and Refining } \\
\text { American Smelting and Refining }\end{array}$ \\
\hline $\begin{array}{l}\text { Minerai de fer } \\
\text { Iron Ore Co. of Canada Ltd. (1954) }\end{array}$ & $\begin{array}{r}52 \\
5\end{array}$ & $\begin{array}{l}\text { Hanna Mining } \\
\text { Hollinger Mines } \\
\text { Bethleem Steel } \\
\text { Republic Steel } \\
\text { National Steel } \\
\text { Wheeling Pittsburg Steel } \\
\text { Armco Steel } \\
\text { Youngstown Sheet and Tube } \\
\text { U.S. Steel Corp. } \\
\text { Steel Co. of Canada }\end{array}$ \\
\hline \multicolumn{3}{|c|}{$\begin{array}{l}\text { a. } \quad \text { L'Asbestos Corp. fut achetée par le gouvernement du Québec en } 1979 . \\
*:\end{array}$} \\
\hline
\end{tabular}

exploités permit l'organisation d'ensembles industriels nationaux qui allaient assurer la transformation primaire et secondaire des minerais ${ }^{32}$. Une entreprise, la Noranda Mines Ltd., contrôlée par des intérêts financiers ontariens, joua un rôle entrepreneuriel de premier plan dans le secteur, et dans la région, dès son organisation en 1922. L'importance de cette entreprise découla, d'une part, de la richesse des gisements initiaux qu'elle possédait - ils contenaient une importante concentration d'or - et, d'autre part, du contrôle indirect qu'elle exerçait sur les entreprises minières avoisinantes du fait qu'elle possédait une importante fonderie de cuivre ${ }^{33}$. La Noranda Mines devint ainsi l'une des

\footnotetext{
32 L'organisation et l'expansion de l'industrie du minerai de cuivre au Québec, dont celle du nord-ouest québécois, sont examinées en plus grands détails dans Paquette, op . cit., 224-269.

${ }_{33}$ En 1930 , par exemple, la mine Horne comptait pour $94 \%$ et $83 \%$ respectivement de la production de cuivre et d'or du Québec; entre 1937 et 1946, il fut extrait de la mine, pour le plus
} 
plus importantes entreprises minières non seulement au niveau national mais aussi au niveau mondial ${ }^{34}$.

Le principal facteur qui justifia l'appui donné à l'industrie minière par les différents gouvernements qui se succédèrent à Québec fut la conviction, maintes et maintes fois répétée, que l'organisation et l'expansion de l'industrie minière étaient pour générer d'importants avantages économiques à l'ensemble de la collectivité. Cette attitude ressort nettement des déclarations de presque tous les responsables du secteur minier depuis 1880 , année de l'adoption de l'Acte général des mines de Québec, pièce maîtresse de la législation minière québécoise ${ }^{35}$. Ces espoirs furent-ils réalisés? La réponse à cette question requiert une évaluation des retombées de l'industrie. Tous les bénéfices économiques potentiels découlant d'une activité économique quelconque peuvent être associés aux différents effets d'entraînement que l'on retrouve dans la théorie du développement économique ${ }^{36}$. Nous en examinerons deux: l'effet aval et l'effet amont. Notre principale conclusion est que ceuxci ont été relativement faibles au Québec; cette constatation, qui s'explique en bonne partie par la satellisation de l'industrie minière, suggère fortement, en conséquence, que l'espoir qu'un développement industriel important accompagnerait l'expansion du secteur s'est avéré, en général, très mal fondé. Nous examinerons tout particulièrement l'effet

grand bénéfice des actionnaires, 78,1 tonnes d'or et 361483 tonnes de cuivre; durant la période 1926-1945, les bénéfices annuels moyens nets furent de 7,3\$ millions; ils s'élevaient à $5 \$$ millions en moyenne entre 1928-37 et à près de $10 \$$ millions entre 1938-47. La fonderie de Noranda, construite en 1922, comptait, en 1968 , pour $85 \%$ de la capacité totale des fonderies de cuivre du Québec et $21 \%$ de la capacité totale canadienne. Sources: Canadian Minerals Yearbook (1968); Rapport annuel du service des mines de Québec (Québec, 1935), 52, 53; L. Roberts, Noranda (Toronto, 1956), 116; cette dernière possède tous les attributs d'une histoire officielle de l'entreprise.

34 En 1965, les exploitations minières qu'elle contrôlait totalisaient $54 \%$ de la capacité totale des moulins de transformation primaire du Québec; en 1975, la proportion atteignait $70 \%$. En 1973, la Noranda contrôlait 19 compagnies minières, réparties dans cinq provinces et dans quatre pays, et 22 entreprises manufacturières appartenant à sept secteurs économiques différentes. Sources: Canadian Mines Handbook; Financial Post Survey of Mines et Noranda Mines $L t d$, Financial Post Corporation Service, années indiquées.

35 Les déclarations ministérielles, à l'occasion de la publication de rapports et de la présentation de mesures législatives, nous ont fourni la principale source d'explication des objectifs de la politique minière au Québec. Nous y revenons dans la troisième partie.

36 Le concept d'effet d'entraînement réfère à la capacité que possède une activité économique donnée d'engendrer des activités économiques secondaires. L'économiste Albert Hirschman est à l'origine d'une nomenclature formelle. Voir A.O. Hirschman, The Strategy of Economic Development (Yale University Press, 1958), chap. 6, et du même auteur, «A Generalized Linkage Approach to Development with Special Reference to Staples», dans Economic Development and Cultural Change, Vol. 25, Supplément, 1977. Les principaux types d'effets d'entraînement se retrouvent dans la théorie des produits de base de la croissance économique; cette dernière, formulée de façon la plus complète par l'économiste M.H. Watkins, stipule que la croissance des économies exportatrices de matières premières peut résulter d'un processus de diversification économique autour des secteurs primaires. Cette théorie, ou approche, a été appliquée par l'auteur dans une étude plus complète des effets d'entraînement générés par le secteur minier québécois; voir Paquette, op. cit., 24-53, et 330-504. Pour l'énoncé «classique» de la «Staple Theory», voir M.H. Watkins, «A Staphe Theory of Economic Growth», dans Canadian Journal of Economics and Political Science (mai 1963). 
aval car c'était à ce niveau que les retombées économiques escomptées étaient les plus importantes.

L'effet d'entraînement en amont réfère aux activités mises en place en vue de fournir des intrants à un secteur donné; son importance dépend, en grande partie, de la nature des technologies qui y sont utilisées; l'usage d'une technologie intensive en capital, par exemple, par opposition à une technologie intensive en travail, laisse entrevoir d'importantes retombées au niveau de la fabrication d'outillage et d'équipements lourds utilisés dans l'extraction. Â cet égard, il est clair que la substitution, au tournant du siècle, des technologies d'extraction de type sélectif, faisant appel à une main-d'oeuvre abondante, en faveur de celles de type non sélectif, utilisant un capital fixe plus important, élargissait considérablement le potentiel d'industrialisation via l'établissement de liens industriels de type amont. Plusieurs études concluent cependant à la faiblesse de l'effet amont sur l'ensemble du $\mathrm{XX}^{\mathrm{e}}$ siècle; l'économiste Gibson, par exemple, notait en 1956 que le Canada demeurait durant la première décennie de l'après-guerre, aussi dépendant des États-Unis pour l'obtention d'équipements miniers qu'il l'était dans les années 1920; en 1970, une étude québécoise sur le sujet confirmait la faiblesse de l'effet amont, soulignant que la «quasi-totalité» des fournisseurs de l'industrie étaient localisés à l'extérieur du Québec ${ }^{37}$.

Deux facteurs principaux expliquent la faiblesse de l'effet amont. Il y a tout d'abord la carence historique du Québec dans le domaine de la fabrication d'équipements industriels; cette carence, qui s'aggrava même après la guerre, maintenait à des niveaux très élevés la propension à importer la machinerie et les équipements miniers au Québec; l'expansion du secteur minier après 1945 ne modifia pas cette situation ${ }^{38}$. Le second facteur est la propriété non québécoise de l'industrie minière; nous avons déjà indiqué l'importance de la propriété étrangère dans les secteurs de l'amiante et du minerai de fer; pour l'ensemble de l'indus-

\footnotetext{
37 Voir J.D. Gibson, «The Changing Influence of the United States on the Canadian Economy», dans Canadian Journal of Economics and Political Science (novembre 1956); l'étude québécoise est de P. Tardivier, Etude sur les problèmes récents de l'industrie minière (nonpubliée), (Ministère des richesses naturelles, Québec, 1970), 8. L'effet en amont est examiné de façon plus détaillée dans Paquette, op. cit., 380-406.

38 La détérioration du Québec dans ce domaine est indiquée par la chute de $33 \%$ à $21 \%$ de la part québécoise de la production canadienne de machinerie industrielle entre 1945 et 1975; celle de l'Ontario augmenta de $56 \%$ à 64\%. Sources: Industries manufacturières du Canada, Statistique Canada, \# cat., 31-203, 31-205, 31-206. Des données précises sur les importations sont plutôt rares; par ailleurs Nickel et al calculaient qu'en 1971 environ la moitié des achats canadiens de machinerie industrielle était effectuée à l'étranger; le pourcentage applicable à l'industrie minière cependant est probablement plus élevé car une étude réalisée par l'économiste Stahl indiquait qu'en 1973 les achats intermédiaires locaux de l'industrie minière canadienne équivalaient à seulement $25 \%$ de la valeur de la production de l'industrie, comparativement à $50 \%$ pour les 15 autres secteurs examinés. Ces données suggèrent donc des propensions à importer, de l'industrie minière, plus élevées que 50\%. Voir J. Stahl, Aggregate Impacts of the Mineral Industry on the Canadian Economy (Énergie, Mines et Ressources, Ottawa, 1973), 9, cité dans P.E. Nickel, et al, Economic Impacts and Linkages of the Canadian Mining Industry (Kingston, 1978), 36 et le tableau 30 , 116.
} 
trie minière, nous pouvons fixer le degré de contrôle non québécois à environ $80 \%$ pour les années $1970^{39}$. Ce facteur tire sa pertinence du fait que l'entreprise sous contrôle étranger est caractérisée par une propension à importer, qui est plus élevée que l'entreprise sous contrôle national ou local ${ }^{40}$; les travaux de Wilkinson, par exemple, font ressortir l'existence d'un lien direct entre le degré de propriété étrangère et les propensions à importer ${ }^{41}$. La satellisation de l'industrie aurait donc été un obstacle à des effets amont plus importants.

Le second effet d'entraînement que nous avons jugé utile d'examiner ici est l'effet aval; celui-ci réfère essentiellement au développement des diverses filières industrielles associées aux principaux minerais exploités ${ }^{42}$. Les effets aval potentiels reliés à l'extraction minière paraissent, en principe, très importants. En effet, l'extraction de minerais constitue la première des multiples étapes associées à leur transformation et à leur fabrication; une valeur ajoutée plus élevée est aussi observée aux étapes plus avancées de chacune de ces filières. Il est possible de jauger l'ampleur possible des effets aval en identifiant les industries de chaque filière qui sont dépendantes des ressources, c'està-dire qui obtiennent un pourcentage important, $50 \%$ par exemple, de leurs intrants du secteur primaire. L'importance des matières premières dans la structure des coûts créerait, pour ces secteurs, des avantages «naturels» de localisation à proximité des ressources. De tels critères permettent de déceler les industries manufacturières, primaires et secondaires, qui, pour des raisons de coûts, auraient tendance à s'établir localement en aval des industries minières correspondantes; l'effet aval potentiel serait important là où la dépendance sur les ressources est

39 Vu l'état des données à cet égard, ce pourcentage est approximatif. Le degré de propriété non canadienne du secteur minier québécois s'établissait à près de $60 \%$ entre 1969 et 1977; la moyenne canadienne s'élevait à $46 \%$. Tenant compte du fait que d'importants secteurs de l'industrie ont été contrôlés de l'Ontario (cuivre, or, zinc), nous pouvons fixer à $80 \%$ son degré de propriété non québécoise. Les données sur la propriété non canadienne sont transposées de celles sur la répartition du revenu imposable des entreprises minières au Canada (minéraux combustibles exclus) selon la localisation (étrangère ou canadienne) du contrôle majoritaire. Sources: Loi sur les déclarations des corporations et des syndicats ouvriers, (Ministère de l'Industrie et du Commerce, Ottawa, années diverses).

40 Pour les annnées 1960, par exemple, il fut calculé que 34\% des grandes entreprises sous contrôle étranger importaient plus de $20 \%$ de leurs achats, contre seulement $5 \%$ des grandes entreprises contrôlées localement. Sources: Gouvernement du Canada, Investissements étrangers directs au Canada, (Ottawa, 1972), 215; les données de cette étude sont tirées de A.E. Safarian, Foreign Ownership of Canadian Industry (Toronto, 1966).

41 B.W. Wilkinson, Canada's International Trade: An Analysis of Recent Trends and Patterns (The Canadian Trade Committee et Private Planning Association of Canada, Montréal, 1968), 145-150. Les raisons des propensions plus élevées des entreprises étrangères sont aussi examinées dans Gouvernement du Canada, op. cit., 217-225.

${ }_{42}$ Une description des filières industrielles reliées à l'extraction de l'amiante, du cuivre et du minerai de fer se trouve dans Paquette, op. cit., 413-414, 449-450 et 479-480; l'Annuaire des minéraux du Canada, une publication annuelle d'Énergie, Mines et Ressources, recense les principaux usages de ces minerais. 
forte ${ }^{43}$. Sept industries pan-canadiennes ont été identifiées comme étant fortement liées, ou dépendantes, soit à l'industrie minière (dépendance directe), soit à l'industrie de transformation primaire (dépendance indirecte $)^{44}$; ces industries sont celles du fer et de l'acier, de la fonte et de l'affinage, de l'estampage, du laminage et du moulage des métaux, de la fabrication de tubes et de tuyaux d'acier, de la production de charpentes métalliques, et de l'usinage de fils métalliques et de produits connexes ${ }^{45}$. Plusieurs de ces activités font partie des filières industrielles associées aux trois principaux minerais exploités au Québec. L'évaluation de l'effet aval se résume donc à examiner leur importance; il s'agit de savoir dans quelle mesure les étapes dépendantes des ressources sont apparues suite à l'expansion de l'extraction ${ }^{46}$.

L'étude des filières industrielles des trois plus importants secteurs de l'industrie minière indique que, dans deux d'entre eux, l'effet aval fut relativement faible. Malgré le fait que plusieurs industries de leurs filières soient, d'après les critères examinés précédemment, dépendantes des ressources, les effets aval associés à l'exploitation du minerai de fer et de l'amiante furent presque nuls ${ }^{47}$. Vu l'importance historique de la sidérurgie dans le processus d'industrialisation, la faiblesse de la filière sidérurgique fut particulièrement désavantageuse. Ce qui est tout à fait paradoxal au sujet de ce secteur, c'est que l'expansion de l'extraction du minerai de fer après 1950 coïncide avec une accentuation du déséquilibre qui s'était formé au début du siècle entre la production et la consommation d'acier au Québec. Entre 1955 et 1975 par exemple, le Québec compta pour environ $25 \%$ de la production cana-

43 Deux études canadiennes ont fait usage de ces critères; voir M.W. Bucovetsky et C.P. Cohen, A Study of the Role of the Resource Industries in the Canadian Economy (Université de Toronto, 1973) et W.D. Bennett, «Les sommes consacrées à l'activité scientifique et l'apport du secteur primaire à l'économie canadienne», dans Études sur certains aspects de la politique des richesses naturelles, Étude spéciale \#27 (Conseil des sciences du Canada, septembre 1973); Bucovetsky fixe à $30 \%$ la part des intrants qu'un secteur doit obtenir du secteur primaire afin d'être qualifié de «dépendant des ressources»; Bennett la fixe à 50\%.

44 La dépendance des ressources est directe si un secteur donné, le secteur A par exemple, obtient du secteur B, un secteur primaire, $50 \%$ ou plus de ses intrants; elle est indirecte si un secteur C obtient $50 \%$ ou plus de ses intrants du secteur B, qui, lui, entretient des liens de dépendance directe avec $\mathrm{A}$.

45 Bennett, op. cit., 19.

46 Il est évident qu'un secteur industriel local en principe dépendant des ressources pourrait se développer tout en étant alimenté de matières premières importées; dans tous les cas, l'essor de filières industrielles repose d'abord et avant tout sur l'apparition d'une demande suffisante pour les biens en cause.

${ }_{47}$ Presque toutes les industries de la filière sidérurgique sont dépendantes des ressources; quatre des sept secteurs énumérés plus tôt font partie de celle-ci; plusieurs des industries de la filière de l'amiante peuvent aussi être qualifiées de dépendantes des ressources; c'est le cas du groupe des produits tissés, des matériaux de construction (e.g. produits isolants, linoleums) et des matériaux de friction (e.g. joints et garnitures). La faiblesse de la filière industrielle de l'amiante est aussi indiquée par le fort taux d'exportation: plus de $95 \%$ sur l'ensemble du $\mathrm{XX}^{\mathrm{e}}$ siècle. En 1925 et 1930 , le nombre de travailleurs dans les mines d'amiante était 10 fois plus élevé que ceux oeuvrant à sa fabrication; en 1969, la proportion était de 5 pour 1 . Sources: Industries manufacturières du Canada, op. cit., Manufacturiers de produits en amiante, Statistique Canada \# cat. 44-203 (années diverses), Manufactures of the Non-metallic Minerals in Canada, \# cat. 44-D22. 
dienne de minerai de fer, soit plus que l'Ontario, mais durant cette même période, la part québécoise de la consommation d'acier fut moindre, se situant à $20 \%$, tandis que sa part dans la capacité totale des fours à acier gravita à des niveaux inférieurs à $10 \%{ }^{48}$. Cette faiblesse historique de la sidérurgie primaire bloqua l'expansion de la sidérurgie secondaire; elle freina aussi l'expansion des filières industrielles du cuivre et de l'amiante ${ }^{49}$.

L'effet aval relié à l'expansion de l'industrie de l'extraction du cuivre contraste avec ceux de l'amiante et du minerai de fer. L'expansion de l'extraction fut dans ce cas accompagnée d'un essor équivalent de l'ensemble des industries de la filière industrielle cuprifère, dont le raffinage et la fabrication de produits de cuivre finis et semi-finis ${ }^{50}$. L'ensemble de la filière du cuivre au Québec fut en fait jugée, dans les années 1970, comme étant la plus complète des grappes industrielles québécoises, tant par ses effets amont qu'aval ${ }^{51}$.

Deux facteurs complémentaires expliquent, selon nous, la présence d'un important effet aval dans le secteur cuprifère et son absence quasi-totale dans ceux de l'amiante et du minerai de fer. Le premier fut

48 Le déclin de la sidérurgie au Québec fut par ailleurs accompagné d'un renforcement de ce secteur en Ontario; sources des données: Industries manufacturières du Canada, op. cit. (années diverses), Primary Iron and Steel et Iron and Steel Mills, Statistique Canada, \# cat. 41-203 (années diverses) et L'Acier au Québec (Ministère de l'Industrie et du Commerce, Québec, 1980), 46,77 . Soulignons que la faiblesse de l'acier primaire ralentit l'expansion de la filière dans son ensemble; par exemple, entre 1955 et 1975 , la part québécoise de la production canadienne de machinerie industrielle chuta de $32 \%$ à $22 \%$, celle des produits électriques baissa de $26 \%$ à $24 \%$ et celle du matériel de transport déclina de $14 \%$ à $11 \%$; tous ces secteurs sont d'importants consommateurs d'acier primaire; sources des données: idem.

49 Le fait que la fabrication secondaire du cuivre ait été plus faible, durant la période de l'après-guerre, que la fabrication primaire est imputable, du moins en bonne partie, à l'absence d'un secteur sidérurgique primaire important au Québec; le fer et l'acier primaire constituent, par exemple, pour le secteur de la fabrication des produits électriques, des intrants aussi importants que le cuivre; en 1966 ceux-ci comptaient pour $11 \%$ des intrants de ce secteur; Sources: «Le système de comptabilité économique du Québec (1966)», Statistiques (juin 1972). Rappelons ici que plusieurs auteurs ont démontré que l'industrie ayant le plus fort volume d'effets d'entraînement était celle du fer et de l'acier, c'est-à-dire la sidérurgie primaire; d'autres ont souligné l'attrait important de ce secteur dans la localisation des secteurs manufacturiers lourds; voir en particulier, S. Czamanski, Study of Clustering of Industries et Study of Spatial Industrial Complexes (Halifax, 1974, et 1976) et H.B. Chenery et T. Watanabe, «International Comparisons of the Structure of Production», texte présenté à la réunion annuelle de la Econometric Society (décembre 1956).

50 Très peu de cuivre québécois a été exporté à l'état brut; déjà en 1945, le rapport entre la production de cuivre raffiné et de cuivre brut s'élevait à 96\%. Source: Annual Report on the Mineral Production of Canada (Ottawa, 1945). Par ailleurs, la disponibilité de cuivre raffiné soutena l'industrie de la fabrication du cuivre; la période de l'après-guerre fut témoin, par exemple, de l'expansion marquée de l'industrie québécoise des câbles et des fils électriques, un secteur dépendant des ressources; la part du Québec dans la production canadienne de cette industrie gravita autour de $45 \%$; celle de la fabrication primaire (laminage, moulage, extrusion, et produits du cuivre) se situa à près de $30 \%$. Sources: Fabricants de fils et de câbles électriques, Statistique Canada, \# cat. 43-209, et Laminage, moulage et extrusion du cuivre et des alliages de cuivre, \# cat. 41-224 (années diverses).

51 Une étude statistique utilisant un tableau des relations intersectorielles de l'économie du Québec identifiait la fonte et l'affinage du cuivre comme étant le secteur-clé de l'économie québécoise; voir M. Boucher, «Identification des secteurs-clés de l'économie québécoise», Revue statistique du Québec (déc. 1974): XXXI. 
la propriété étrangère de ces deux industries et le second fut leur intégration totale aux étapes les plus avancées des filières industrielles situées à l'extérieur du Québec. En effet, ainsi que nous l'avons déjà signalé, ces deux secteurs d'extraction passèrent très tôt aux mains du capital américain et furent intégrés aux structures oligopolistiques mondiales et nord-américaines de l'industrie minière, de même qu'aux structures oligopolistiques parallèles des secteurs manufacturiers correspondants. L'une des conséquences fut que la transformation des matières premières se réalisa dans le contexte des stratégies globales des entreprises manufacturières impliquées; les stratégies de mise en marché et de localisation des installations de transformation visèrent d'abord à approvisionner les marchés les plus importants à partir d'installations de fabrication déjà établies, les marchés jugés secondaires, tels ceux du Québec, étant subséquemment desservis à partir de celles-ci. Ces stratégies contribuèrent cependant à perpétuer la sous-industrialisation de l'économie québécoise, et cela, même si les seuils de rentabilité dans la transformation de ces deux minerais avaient, en principe, été atteints au moment où débuta l'extraction, ou peu après ${ }^{52}$. Les régimes nordaméricains de fixation des prix de ces deux minerais eurent aussi le même effet car ils représentaient d'autres barrières, artificielles, mais non moins réelles, à la transformation locale des minerais ${ }^{53}$. Ces barrières bloquaient l'organisation de nouvelles entreprises manufacturières et perpétuaient, en conséquence, le pouvoir de marché des entreprises intégrées déjà établies.

L'examen des effets aval suggère qu'il y eut un lien important entre la structure de développement des secteurs de l'amiante et du minerai de fer et leurs structures de propriété; ces dernières menèrent à leur satellisation, ce qui explique, en grande partie, la faiblesse de leurs apports économiques. Quant au secteur cuprifère, celui-ci constitue un exemple où les avantages «naturels» de la proximité des ressources jouèrent pleinement. Ce secteur ne fut pas développé, ni intégré, par des complexes manufacturiers déjà en opération; la disponibilité du capital financier anglo-canadien, la disponibilité d'électricité et de maind'oeuvre, et finalement, la disponibilité d'une technologie de transfor-

\footnotetext{
52 Sur la question de seuils de rentabilité, voir Asbestos, Report of the Task Force on Upgrading (Ministère de l'Industrie et du Commerce, Ottawa, juin 1974); en ce qui touche le minerai de fer, voir The Canadian Primary Iron and Steel Industry, Commission royale d'enquête sur les perspectives économiques canadiennes (Ottawa, 1956), 49 et Jacques St-Laurent, «Facteurs de localisation dans l'industrie sidérurgique», dans A. Raynauld, Croissance et structures économiques de la province de Québec (Québec, 1957), 358.

53 Le régime des prix du minerai de fer est bien décrit dans L.G. Hines, «Price Determination in the Lake Erie Iron Ore Market», American Economic Review (septembre 1951); voir aussi N.I. Greenwald, «Supply Shifts and Iron Ore Pricing», dans Journal of Industrial Economics (avril 1961). Une étude sur l'amiante suggère aussi l'existence d'un régime de prix pour la fibre favorable aux producteurs américains de produits d'amiante; voir N. Alexandre, Vers une politique québécoise de l'amiante (Ministère des Richesses naturelles, Québec, 1975), 89.
} 
mation et d'affinage favorisèrent tout l'établissement d'une filière industrielle élaborée.

\section{La politique minière et le renforcement de la satellisation}

La politique du développement minier au Québec fut conçue dans le plus pur des styles du libéralisme et du laisser-faire économiques. L'Acte général des mines de Québec, adopté en 1880 par le gouvernement conservateur d'Adolphe Chapleau, constitue la première mesure législative s'appliquant à tout le secteur minier; depuis, elle s'est avérée être la pierre angulaire de l'ensemble des politiques minières du Québec.

L'Acte général des mines appliquait à l'ensemble du secteur minier le principe de la divisibilité des droits de propriété; en vertu de ce principe, aussi appelé principe régalien, le domaine minier forme un bien distinct de la surface du sol de sorte que la cession des droits superficiaires, par vente ou location, n'entraîne pas la cession des droits de propriété sur les minerais qui pourraient s'y trouver ${ }^{54}$; ceux-ci reviennent à l'autorité politique souveraine, c'est-à-dire à la Couronne ou à l'État ${ }^{55}$. L'application de ce principe par les autorités provinciales constituait ni plus ni moins qu'une extension des pratiques de la période coloniale. Toutes les concessions de terre pendant le Régime français s'effectuèrent sous réserve des droits miniers: «... le fonds appartient au seigneur, mais le tréfonds est au roi», nous rappelle Trudel ${ }^{56}$; la cession des droits miniers devait faire l'objet d'un octroi spécifique, qui, habituellement, réservait à la Couronne un droit régalien fixé à un dixième de la production ${ }^{57}$.

À l'époque du Régime anglais, les pratiques donnèrent lieu aussi, malgré plusieurs divergences avec le Régime qui le précédait, dont, par exemple, la tenure en franc et commun soccage, à l'application du

54 L'amendement de 1884 de la loi générale expliquait ainsi le principe de la divisibilité; voir «Acte pour amender de nouveau l'acte général des mines de Québec de 1880», 47 vict., chap. 22, art. 1, Statuts de la province de Québec (1884).

55 Un autre principe régissait la même question: celui de l'indivisibilité, ou régime d'acquisition (acession system), en vertu duquel la disposition du domaine public à des fins agricoles, par exemple, entraînait automatiquement la cession des droits de propriété sur les ressources minérales qui pourraient, ultérieurement, y être localisées. Une discussion plus élaborée du fondement historique des deux principes se trouve dans Paquette, op. cit., 91-98.

56 M. Trudel, Le régime seigneurial (Ottawa, 1971), 15. L'ambiguité, sur cette question de certains titres seigneuriaux fut l'objet d'importants litiges au milieu du XIX ${ }^{\mathrm{e}}$ siècle; il fallait spécifier le sens exact de la formule «donner avis des mines au roi»; en 1883, la Cour d'appel opina que cette formule équivalait effectivement à réserver les droits miniers au profit de la Couronne; l'un des litiges se rapportait à des contestations de droits à l'intérieur de la seigneurie Rigaud-Vaudreuil; voir Bouffard, Traité du Domaine (Québec, 1921), 131; ces questions sont aussi traitées dans Procès DeLery, factums pour et contre (Québec, 1868) et dans Rapports judiciaires de Québec, 9, Régina v. DeLery et al (1883): 225, 247.

57 A.M. Hart, Practical Suggestions on Mining Rights and Privileges in Canada (John Lovell, Montréal, 1867), 14. Pour de plus amples détails à l'égard des pratiques sous le Régime français, voir Paquette, op. cit., 99-103. 
principe de la divisibilité ${ }^{58}$; cela était d'ailleurs conforme tant aux politiques de l'empire qu'aux traditions anglaises ${ }^{59}$. L'intensification des activités minières qui se produisit après le milieu du XIX ${ }^{\mathrm{e}}$ siècle au Québec offrit ainsi l'occasion de faire connaître ce principe de façon explicite. Un ensemble de lois et de règlements furent donc promulgués à cette fin, fixant, entre autres choses, le mode d'aliénation du domaine minier sur les terres publiques et privées à des fins de recherches minières et d'exploitation ${ }^{60}$. Ces pièces législatives, tels «l'Acte concernant les mines d'or» de 1864 et «l'Acte concernant la vente de terrains pour l'exploitation des mines de phosphate», adopté en 1878, définissaient également les conditions en vertu desquelles les droits miniers étaient cédés $^{61}$. Par exemple, la vente de concessions minières de superficies régulières et à prix fixe date de cette époque; le versement de redevances minières était également prévu ${ }^{62}$. C'est cette législation que la loi générale de 1880 étendait à tous les minerais que pouvait receler le sous-sol québécois ${ }^{63}$.

Dans ses deux parties principales, celle ayant trait au mode d'aliénation du domaine minier et celle touchant les redevances, la législation minière de 1880 fut incitatrice et visait à encourager l'expansion maximale d'une industrie qui, jusqu'alors, avait été plutôt marginale, mais qui était néanmoins susceptible de fournir des emplois. L'honorable $\mathrm{E}$. Flynn, commissaire des terres de la Couronne, justifia sa politique de la façon suivante:

Chaque mine ouverte devient, on le sait, un marché pour les produits du sol et de la ferme; ...

\footnotetext{
58 Bouffard, op. cit., 142-144.

59 Voir P.T. Flawn, Mineral Resources (Chicago, 1966), 155-156 et T.A. Rickard, Man and Metals (New-York, 1932), 600-606.

60 Voir A. Leroy, Les mines de Québec (Québec, 1906), 72-73.

61 27-28 Vict., chap. 9, Statuts du Canada, 1864 et 41 Vict. chap. 4, Statuts de la province de Québec, 1878.

62 Cela était aussi conforme aux pratiques à l'intérieur de l'Empire britannique; des droits régaliens, ou redevances, de $20 \%$ étaient prévus sur la production de métaux précieux lors de la cession de droits par la Couronne; dans l'empire colonial espagnol, ce droit régalien, aussi présent, s'appelait le «quinto"; de nombreux exemples se trouvent dans A. Del Mar, A History of the Precious Metals, (2e édition, New-York, 1968), 229-230, 25-49, 368, 391.

${ }_{3}$ Voir 43-44 Vict., chap. 12, Statuts de la province de Québec (1880); en vertu de cette loi, le principe de la divisibilité ne s'appliquait pas aux terres concédées avant 1880; cette disposition fut annulée en 1890 par le gouvernement libéral d'Honoré Mercier au grand désespoir des opérateurs de l'industrie qui demandèrent au gouvernement fédéral de désavouer la loi; cette modification fut cependant abrogée lorsque le parti conservateur forma un nouveau gouvernement. Le principe de la divisibilité ne s'appliqua donc pas aux régions d'amiante des Cantons de l'est car les terres en cause avaient été octroyées par la Couronne avant 1880; sur ces terres, seul l'or, l'argent et le phosphate étaient réservés à la Couronne. Voir Bouffard, op. cit., 128-140, 147; Leroy, op. cit., 79-81; la loi adoptée en 1890 par le gouvernement libéral fut «l'Acte pour amender et refondre la loi des mines de $1880 », 54$ Vict., chap. 15, tandis que celle qui abrogeait celle-ci fut «l'Acte pour amender et refondre la loi des mines», 55-56 Vict., chap. 22, Statuts de la province de Québec (1890 et 1892).
} 
$\ldots$ ouvrons les portes toutes grandes aux mineurs de tous les pays; ils vont accourir ici et faire surgir du sol des richesses et des fortunes qui serviront à leur tour à développer d'autres industries. ${ }^{64}$

Le mode d'aliénation retenu fut la vente de concessions au lieu de la location de droits miniers. Dans l'optique de l'exploitant ou du prospecteur, la vente accordait une bien meilleure sécurité quant à la durée des droits octroyés par l'État; c'est pour cette raison, d'ailleurs, que ce mode fut adopté, et ce, contrairement au secteur forestier où le mode locatif était à l'honneur ${ }^{65}$. Selon le Commissaire Flynn, il fallait «faciliter l'obtention des permis et donner à ceux qui transigent avec nous parfaite sûreté à tous les points de vue» ${ }^{66}$. Il fallait surtout, à l'époque, rassurer les «capitalistes étrangers» qui s'apprêtaient à investir dans le secteur aurifère ${ }^{67}$. Les charges financières furent donc prescrites dans cet esprit. Une double structure de prix fut retenue pour la vente de concessions: 2,00\$ l'acre sur les terres aurifères et argentifères et 1,00\$ l'acre pour les autres minéraux, soit un prix identique aux terres vendues pour les fins de l'agriculture ${ }^{68}$. Un régime libéral de jalonnement de concessions et de permis d'exploitation et de prospection fut également généralisé par la législation de 1880; celle-ci prévoyait même qu'en cas de mésentente entre un propriétaire superficiaire et un détenteur des droits miniers, ce dernier pouvait exiger que le propriétaire de la surface du sol soit exproprié69. Quant aux redevances, la loi de 1880 les imposait, en principe, seulement à l'or, à l'argent et au phosphate, mais laissait leur perception à l'entière discrétion du gouvernement ${ }^{70}$. Afin de ne pas «mettre en péril l'industrie minière encore à l'état d'enfance», il fut décidé cependant de ne pas en exiger ${ }^{71}$. Si le trésor public fut sacrifié par ce choix, la communauté minière, par ailleurs, voyait ses attentes entièrement comblées; celles-ci étaient résumées ainsi par le Morning Chronicle de Québec peu après l'adoption de la loi générale:

64 Débats de l'Assemblée législative du Québec (20 juin 1890); 539-40, 544.

65 Ce fut la Crown Timber Act de 1849 qui fixait le mode d'aliénation des ressources forestières; le paiement de rentes et de droits de coupe fut prévu de même que l'octroi de droits de propriété via le mode locatif. Voir Hamelin et Roby, op. cit., 208 et A.R.M. Lower, «Settlement and the Forest Frontier in Eastern Canada», dans W.A. Mackintosh, et W.L.G. Joerg, op. cit., 76-77. Le caractère renouvelable des ressources et la rivalité entre l'agriculture et l'exploitation forestière aident à expliquer la prédominance du mode locatif d'aliénation des droits dans le secteur forestier; une analyse comparée des pratiques dans les deux secteurs serait sans doute fort intéressante.

66 Débats de l'Assemblée législative du Québec (14 juin 1892): 250.

67 Ibid. (13 juillet 1880): 654. Rappelons que le principe de la divisibilité n'était pas appliqué aux U.S.A.; voir à ce sujet, Flawn, op. cit., 160 et E. Northcutt, «Mineral Titles and Concessions», dans E.H. Robie, Economics of the Mineral Industries (New-York, 1964), 100-102, 106.

68 Voir 43-44 Vict., chap. 12, articles 21-35 et Documents de la session, A1870, no 2, appendice 24 et A1874, no 2, appendice 49.

69 Voir 43-44 Vict., chap. 12, articles 21-22, 47, 50, 76, 82; pour une description plus approfondie des régimes en cause, voir Paquette, op. cit., 118-127.

43-44 Vict., chap. 12, art. 13.

71 Débats de l'Assemblée législative du Québec (14 juin 1892): 246. 
... we are free to say that if the Government err at all at this juncture, it should rather do so on the side of liberality to the mining interest than on any other, in view of the vast future benefits which the healthy development of that important interest under favourable conditions may be confidently expected to confer upon the Province as a whole. ${ }^{72}$

L'opposition libérale de l'époque critiqua sévèrement ce qu'elle voyait comme étant une politique excessivement généreuse à l'endroit des intérêts privés du secteur. Le régime des redevances fut particulièrement mis en cause. C'est pour cette raison qu'à son retour au pouvoir le parti libéral d'Honoré Mercier s'empressa de modifier cette partie de la loi: tous les minerais furent frappés de redevances et leur perception devint une obligation statutaire ${ }^{73}$. Le commissaire Georges Duhamel, parrain des amendements, allégua qu'une libéralité excessive en ce domaine ne pouvait être justifiée: «En premier lieu», dit-il, lors d'un débat sur la question, «la province s'est dessaisie de ces propriétés pour une bagatelle; en second lieu, elle a dépensé des millions dans la construction de chemins de fer, en vue de développer ces propriétés... Or, qu'arrive-t-il aujourd'hui? Ceux qui bénéficient le plus de la prodigalité de ce gouvernement paternel sont les premiers à lui refuser le droit de les taxer et de se dédommager quelque peu des énormes dépenses encourues par le pays pour l'avantage des capitalistes. ${ }^{74}$ Le secteur de l'amiante, le plus important à l'époque, fut identifié de façon particulière à cet égard: «... les mines ont été vendues par le gouvernement à des prix insignifiants à des personnes qui, dans une foule de cas, en ont retiré des bénéfices énormes»; dans certains cas, des coûts d'extraction de «\$30 à \$40 la tonne» se comparaient, fort avantageusement, à des recettes de $\ll 200$ à $\$ 250$ la tonne» ${ }^{75}$. Cette volonté de rééquilibrer la répartition des bénéfices ne put cependant être menée à terme car les conservateurs revinrent au pouvoir peu après l'adoption des amendements libéraux et rétablirent le pouvoir discrétionnaire à l'égard des redevances ${ }^{76}$. Le laxisme dans ce domaine fut même poussé au point où aucune redevance ne fut perçue par la Couronne de 1880 à $1917^{77}$. Cette situation contrasta avec celle de l'Ontario où l'expansion minière du début du siècle fut suivie de l'imposition d'un régime de redevances beaucoup moins libéral ${ }^{78}$.

\footnotetext{
${ }^{72}$ Quebec Morning Chronicle, The Chaudière Valley and its Mineral Wealth (Québec, 1880), 60-61.

77 C'est ce qu'indiquent les documents officiels de la période; voir Rapport annuel (Ministère des terres de la Couronne, 1879 à 1896) et Rapport annuel (Dép. de la colonisation, mines et pêcheries, 1897 à 1917).

78 Entre 1895 et 1915 , par exemple, la part des revenus miniers dans la production minière totale fut de 1,43\% en Ontario; au Québec, elle s'éleva à $0,47 \%$, soit trois fois moins; source: Paquette, op . cit., tableau 6.1:345.
} 
La politique minière québécoise au $\mathrm{XX}^{\mathrm{e}}$ siècle en fut une de continuité. Plusieurs modifications y furent apportées, mais sans que cela ne change son caractère typiquement libéral et incitateur. Le mode d'aliénation des droits miniers retenu en 1880 , la vente de concessions, ne fut éliminé que 85 ans plus tard, en 1965, lorsque fut adopté à sa place un régime de cession des droits par bail, c'est-à-dire le système locatif. Cette substitution survint cependant après que les zones minéralogiques les plus prometteuses eussent été cédées aux anciennes conditions, beaucoup plus favorables ${ }^{79}$. La question des redevances, par ailleurs, fut réexaminée avec plus de diligence. En 1917, le gouvernement de Lomer Gouin imposa une redevance au secteur de l'amiante ${ }^{80}$. En 1925, celui d'Alexandre Taschereau introduisit une échelle de redevances graduée en fonction des bénéfices d'opération des entreprises et l'appliqua à tout le secteur ${ }^{81}$; calqué sur ce qui était en vigueur en Ontario depuis plusieurs décennies ${ }^{82}$, c'est ce type de régime qui a été maintenu jusqu'à nos jours ${ }^{83}$. Globalement cependant, le régime québécois des redevances demeura avantageux et fut plus généreux envers les exploitants que le régime en vigueur dans le secteur forestier ou que celui en force dans l'industrie minière de l'Ontario. Sur l'ensemble du XX $\mathrm{X}^{\mathrm{e}}$ siècle, la part que les revenus miniers représentaient dans la production minière totale fut inférieure à celle de ces deux autres secteurs: $1,83 \%$ en moyenne au Québec par rapport à 1,94\% en Ontario (entre 1895 et 1976) et à 5,79\% dans le secteur forestier (entre 1905 et 1976) ${ }^{84}$; aussi la majeure partie des rentes de ressources, ou des profits excessifs, générées par la production minière, plus des deux tiers par exemple entre 1967 et 1972, échappa au fisc québécois ${ }^{85}$.

La continuité fondamentale de la politique minière, quant à sa philosophie de base, ressort aussi d'un examen des mises à jour législatives successives qui visaient à la rendre compatible avec les changements structurels qui s'étaient opérés dans le secteur. Deux de ces mises à jour en particulier méritent d'être signalées. Il s'agit des modifications aux dispositions législatives du XIX ${ }^{\mathrm{e}}$ siècle à l'égard de l'inactivité des détenteurs de droits miniers, et ensuite, des dispositions par-

79 Ce fut le cas notamment des principaux gîtes localisés dans la fosse du Labrador au Nouveau-Québec; la réforme de 1965 n'était pas d'application rétroactive; voir 13-14 Éliz. 2, chap. 34, Statuts de la province de Québec (1965).

80 Rapport sur les opérations minières de la province de Québec (1920): 16-17.

81 Voir 15 Geo. 6, chap. 27.

82 Rapport sur les opérations minières de la province de Québec (1924): 10; la politique minière de l'Ontario de l'époque est bien décrite dans H.V. Nelles, The Politics of Development ('Toronto, 1975), 160-161, 176.

${ }_{83}$ Des additions d'importance variée y furent apportées en 1935, 1965 et 1976; voir 25-26 Geo. 5, chap. 41, 13-14 Éliz. 2, chap. 35, Statuts de la province de Québec $(1935,1965)$ et Lois d'u Québec (1975): chap. 29 et 30.

84 Des données plus complètes, qui proviennent de sources officielles, et les modes de calculs sont présentés dans Paquette, op. cit., tableau 6.1 et 6.2: 345 et 349 .

85 En principe, ces rentes pouvaient être appropriées au profit de la collectivité sans modifier les niveaux de production; voir sur ces questions, Paquette, op. cit., 356-376. 
ticulières qui furent décrétées à l'égard du territoire du Nouveau-Québec. Rappelons que si la politique minière du XIX ${ }^{\mathrm{e}}$ siècle était avantageuse pour les exploitants, elle leur imposait néanmoins l'obligation de travailler les concessions sur une base continuelle et de débuter l'exploitation le plus rapidement possible. Tout contrevenant risquait la confiscation pure et simple de ses droits. Cette possibilité de confiscation pour cause d'inactivité date de la loi générale de 1880; des amendements en 1892 fixèrent même un niveau minimal de dépenses à effectuer ${ }^{86}$. L'objectif avoué de ces articles de la loi était d'empêcher que l'expansion du secteur minier puisse être freinée par la spéculation. Le commissaire Duhamel fut très explicite à ce sujet:

Par conséquent, l'exploitation minière de la province ne sera plus arrêtée dès le début par ces hommes qui veulent accaparer au moyen de ce système vicieux les richesses minérales du pays, sans aucune intention de les exploiter, mais dans le seul but de les acheter à vil prix du gouvernement et de les revendre à des sommes énormes à ceux qui voudraient les exploiter. ${ }^{87}$

Au XX $\mathrm{X}^{\mathrm{e}}$ siècle, dès 1911 en fait, ces obligations furent assouplies en accordant aux détenteurs de droits miniers la liberté de ne pas effectuer les travaux statutaires en échange du versement de taxes additionnelles au Trésor public ${ }^{88}$; soulignons que ces taxes additionnelles représentaient des sommes moindres que la valeur des travaux statutaires requis $^{89}$. Des amendements subséquents réduisaient un peu plus leur portée en permettant que l'ensemble des travaux statutaires exigés sur plusieurs terrains d'un groupe puissent être effectués sur l'un d'eux. Un amendement en 1924 permettait une telle concentration des travaux sur les concessions tandis que d'autres, en 1930, 1937 et 1965, l'étendaient aux terrains détenus en vertu de permis de mise en valeur et à ceux détenus en vertu d'un bail minier ${ }^{90}$.

Le territoire du Nouveau-Québec couvre une superficie de plus de $300000 \mathrm{~km}$ carrés, soit près de la moitié du territoire québécois. Dès le début du siècle, le potentiel minier de cette région était reconnu comme l'un des plus importants de l'hémisphère occidental; sa zone

\footnotetext{
${ }^{86}$ En 1911, ces dépenses furent spécifiées en nombre de jours ouvrables. Les textes législatifs pertinents sont 43-44 Vict., chap. 12, arts. 34, 87-89; 54 Vict., chap. 15, arts. 1455-1476 et 55-56 Vict., chap. 20, art. 2121; Statuts de la province de Québec (années indiquées).

87 Débats de l'Assemblée législative du Québec (20 décembre 1890): 531

88 Voir 1 Geo. 5, chap. 23, Statuts de la province de Québec (1911).

89 La loi de 1892 fixait les travaux statutaires à 200,00\$ au minimum par lot de 100 acres; en 1911 , ces travaux furent fixés à 25 jours par année sur une concession de 40 acres. La loi de 1911 fixait, par ailleurs, à 1,00\$ l'acre la taxe compensatoire sur les terrains en exploration et à $.10 \varnothing$ l'acre la taxe sur les concessions inactives. L'on dut attendre jusqu'en 1965 pour que les «pénalités» soient augmentées à égalité avec la valeur des travaux requis. Les textes législatifs pertinents sont les suivants: 55-56 Vict. chap. 20,1 Geo. 5, chap. 23,1 Geo. 6, chap. 41 et 13 14 Éliz. 2, chap. 34

Sources: 14 Geo. 5, chap. 31, 20 Geo. 5, chap. 41, 1 Geo. 6, chap. 41 et 13-14 Éliz. 2 , chap. 34
} 
minéralogique fut connue sous le nom de «fosse du Labrador» et elle fut comparée très tôt à la région minière du Mesabi aux États-Unis ${ }^{91}$. $\mathrm{Vu}$ sa relative inaccessibilité cependant, il fut décidé d'assujettir le Nouveau-Québec à une règlementation particulière. Une série d'amendements furent conséquemment apportés à la législation en 1919 afin de suspendre l'application de la loi sur ce territoire et d'accorder au «Gouverneur-général en Conseil», c'est-à-dire le Cabinet des ministres, un pouvoir discrétionnaire en ce qui touchait les conditions en vertu desquelles y étaient cédés les droits aux minéraux ${ }^{92}$. La période de validité des titres émis, de même que la superficie des terrains visés, étaient dorénavant établies par arrêtés en conseil. En 1945, suite à un renouveau d'intérêt dans la région, de nouveaux amendements furent adoptés afin de «faciliter et d'encourager le développement minier» ${ }^{93}$. Tout en renouvelant le pouvoir discrétionnaire du Cabinet d'émettre des permis exclusifs d'exploration, ces amendements l'étendaient à la phase de l'exploitation en conférant aussi au Cabinet la responsabilité d'octroyer des baux d'exploitation à long terme pour tout gisement sur lequel l'extraction commerciale était envisagée ${ }^{94}$. Les conditions qui furent rattachées à l'émission de ces titres furent très généreuses. La superficie moyenne des territoires visés par les permis d'exploration s'éleva à plus de $1500 \mathrm{~km}$ carrés, tandis que celle des zones détenues en vertu d'un bail d'exploitation pouvaient atteindre $450 \mathrm{~km}$ carrés ${ }^{95}$. La période de validité de ces titres était aussi exceptionnelle; un terrain put, par exemple, être détenu en vertu d'un permis d'exploration pour une période allant jusqu'à 15 ans, pourvu seulement que certains travaux fussent effectués chaque année ${ }^{96}$. La période de validité d'un bail d'exploitation pouvait par ailleurs atteindre $80 \mathrm{ans}^{97}$. Les charges financières prévues contrastaient finalement avec celles prélevées sur l'autre moitié du domaine minier québécois. Le permis d'exploration entraînait une charge annuelle équivalant à $50 \phi$ le mille carré tandis que le

91 La fosse du Labrador possède une longueur de $340 \mathrm{~km}$ et une largeur variant de 15 à $90 \mathrm{~km}$; par comparaison, la région du Mesabi, qui fournit pendant plusieurs décennies la majeure partie de la production américaine de minerai de fer était longue de $165 \mathrm{~km}$ et avait une largeur variant entre 1,5 et 7,5km; source: L'Industrie minière de la Province de Québec (1951), 21.

92 Voir 9 Geo. 5, chap. 30, Statuts de la province de Québec (1919).

93 Voir 9 Geo. 6, chap. 54, Statuts de la province de Québec (1945)

94 Des dispositions législatives analogues avaient été adoptées en 1931 à l'égard des puits de pétrole et de gaz naturel, des placers aurifères et de quelques autres minerais recherchés ou exploités sur l'ensemble du territoire; voir 21 Gieo. 5, chap. 45, Statuts de la province de Québec (1931)

Le premier «permis spécial d'exploration» émis en 1919 visait un terrain de $3150 \mathrm{~km}$ carrés; les principaux permis émis entre 1942 et 1953 par arrêtés en conseil visaient des blocs d'une superficie moyenne de $1800 \mathrm{~km}$ carrés; sources: L'Industrie minière de la province de Québec (1946, 1947, 1950, 1953). La superficie des terrains détenus en vertu d'un bail d'exploitation était, en principe, illimitée; la superficie de $450 \mathrm{~km}$ carrés rapportée dans le texte réfère au territoire octroyé à la Hollinger North Share Exploration Co. Ltd. en 1946; voir à cet égard, 10 Geo. 6, chap. 42, Statuts de la province de Québec (1946).

96 Voir 9 Geo. 6, chap. 54; en 1946, le permis de la Hollinger North Shore exigeait des travaux exploratoires d'un peu plus de $10,000 \$$ répartis sur six ans; voir 10 Geo. 6 , chap. 42

97 Ibid., art. 11. 
bail d'exploitation donnait lieu à l'imposition d'une rente annuelle de $100,000 \$^{98}$. Ailleurs, l'étape exploratoire était assujettie à une taxe de $10,00 \$$ par concession (40 acres); une licence d'exploitation coûtait, annuellement, $50 \notin$ l'acre, tandis que l'achat d'une concession nécessitait un déboursé de 3,00\$ l'acre ${ }^{99}$. Le Ministre des Mines de l'époque reconnut explicitement que l'aliénation du domaine minier au Nouveau-Québec allait être réalisé à des «conditions extrêmement faciles»" Celles-ci furent sévèrement critiquées par l'opposition libérale car, selon cette dernière, aucune garantie quant aux retombées n'avait été obtenue en échange ${ }^{101}$.

Les principales mises à jour de la politique minière que nous venons d'examiner avaient deux conséquences principales. Premièrement, elles rendaient inopérantes les dispositions législatives décrétées au XIX ${ }^{\mathrm{e}}$ siècle touchant la révocation de droits aux minéraux pour cause d'inactivité. Deuxièmement, elles favorisaient nettement la monopolisation du domaine minier par les grandes entreprises, et plus particulièrement, par les entreprises intégrées. En pratique, seules les firmes d'une certaine envergure étaient en mesure de détenir des droits sur le territoire du Nouveau-Québec, et une fois en leur possession, ces droits pouvaient être conservés pour de longues périodes de temps. Ces principaux effets des mises à jour signifiaient que l'accès au domaine minier québécois avait été considérablement réduit: un régime de porte close avait presque été institué ${ }^{102}$.

L'objectif officiel des modifications qui furent apportées à la politique minière était évidemment celui de favoriser l'essor de l'industrie et celui de l'économie québécoise. La politique minière sous l'Union nationale de Maurice Duplessis, par exemple, visait à inciter l'établissement éventuel d'une sidérurgie par le secteur privé ${ }^{103}$. Les principales mises à jour cependant furent décrétées dans le contexte global des mutations structurelles majeures, examinées précédemment, de l'industrie minière et manufacturière nord-américaine. La grande entreprise intégrée, souvent de propriété étrangère, était devenue l'entreprise-type de l'industrie minière au $\mathrm{XX}^{\mathrm{e}}$ siècle; c'est ce qui caractérisa

98 Ces charges furent celles imposées en 1946 à la Hollinger North Shore

99 Ces tarifs sont ceux qui étaient en vigueur à la fin de la Deuxième Guerre mondiale lors de l'octroi à la Hollinger North Shore au Nouveau-Québec; en 1950, les prix des concessions passèrent à $9,00 \$$ l'acre et atteignirent $30 \$$ l'acre en 1965 ; source: 14 Geo. 6 , chap. 28 et 13-14 Éliz. 2, chap. 34, Statuts de la province de Québec (1950 et 1965).

100 L'expression apparaît dans une lettre du ministre adressée à Jules Timmins, un actionnaire important de la Hollinger North Shore Exploration Co.; cité dans Le Soleil, 20 mars 1946. 11 .

101 Voir, par exemple, Le Soleil, 13 mars 1946, 11, et le 23 mars 1946, 3.

102 Rappelons que les principaux permis d'exploration émis entre 1942 et 1953 pour le Nouveau-Québec couvraient la totalité de la zone minéralogique favorable de la fosse du Labrador; source: L'industrie minière de la province de Québec (1947, 1950, 1953).

${ }_{103}$ C'est là la justification principale de la libéralité de la politique minière à l'égard du Nouveau-Québec; voir L'Événement, 15 mai 1945, 11, et Le Soleil, 15 mai 1945, 3. 
l'industrie du cuivre, du minerai de fer et de l'amiante au Québec. Ces nouvelles structures oligopolistiques furent aussi marquées par la création de barrières d'entrée aux industries qui en faisaient partie, et par l'élaboration de stratégies corporatives visant à les perpétuer. La création de barrières d'entrée par le biais d'un contrôle des sources d'approvisionnement fut tout particulièrement important à la survie corportive et à une plus grande rentabilité des entreprises consommatrices de matières premières. L'importance de ces barrières est telle que certains auteurs suggèrent même que le contrôle de matières premières explique davantage l'existence d'oligopoles que la présence d'économies d'échelle au niveau des appareils productifs ${ }^{104}$. Une politique minière qui se voulait essentiellement libérale et incitatrice se devait d'être modifiée afin de refléter les nouvelles réalités structurelles de l'industrie et les intérêts des entreprises qui la constituaient. C'est ce qui se produisit. En fait, la nature des mutations structurelles, et la nécessité d'y adapter la politique minière, furent explicitement reconnues vers le milieu du siècle. En 1940, un amendement à la Loi des mines légalisait davantage l'inactivité en accordant aux entreprises productrices le droit de se constituer des réserves de minerai pour exploitation ultérieure ${ }^{105}$. C'était là un renversement complet par rapport à l'approche du XIX siècle et à celle de l'Acte général de 1880 en particulier; entre ces deux périodes cependant, l'industrie minière avait perdu sa structure concurrentielle. Le paradoxe cependant fut de taille: la politique minière québécoise du $\mathrm{XX}^{\mathrm{e}}$ siècle contribua, sans aucun doute, au renforcement des barrières d'entrée à l'industrie minière et aux industries de transformation, et réduisit, en conséquence, l'apport économique général qui découlait de l'expansion des activités extractives.

En créant et en structurant la politique minière, les autorités politiques québécoises identifièrent l'intérêt collectif aux intérêts privés et à ceux du capital étranger qui s'étaient accaparés des principaux secteurs de l'industrie. L'honorable J.-E. Perreault, ministre des Mines

\footnotetext{
104 Voir, par exemple, D. Martin, «Resource Control and Market Power», dans M. Gaffney, ed., Extractive Resources and Taxation (University of Wisconsin Press, Madison, 1967); une étude réalisée pour le compte de la Commission royale d'enquête sur les perspectives économiques canadiennes relevait quelques avantages financiers majeurs à l'intégration verticale en amont; voir J. Davis, Mining and Mineral Processing in Canada (Gouvernement du Canada, 1957), 323, 328.

105 Voir 4 Geo. 6, chap. 28, Statuts de la province de Québec (1940). La «réforme» de 1965 ne modifia pas cet aspect de la réglementation ni les autres changements qui avaient précédemment été apportés à la législation minière. Notons par ailleurs que la fiscalité minière devint de plus en plus favorable aux grandes entreprises intégrées; les «réformes» de 1965 et de 1975, par exemple, redistribuaient le fardeau fiscal des grandes entreprises du secteur aux plus petites, soit les entreprises non intégrées; voir à ce sujet, L'Industrie minière du Québec, 1976 (Ministère des richesses naturelles, 1977): 68; les amendements pertinents sont les suivants: 13-14 Éliz. 2, chap. 35 et Lois du Québec, 1975, chapitres 29 et 30; ces amendements créaient de nouvelles déductions dans le calcul des profits assujettis aux redevances dont bénéficiaient les entreprises minières actives dans la transformation primaire (concentration, fonte ou affinage).
} 
dans le dernier gouvernement d'Alexandre Taschereau, résuma peutêtre le mieux la philosophie libérale qui soutenait la politique minière:

Dans un pays comme le Canada, le gouvernement qui n'accorde pas toute l'assistance possible à ceux qui cherchent à ouvrir la voie à l'exploitation des ressources naturelles ne mérite pas l'appellation de gouvernement. ... le moins que l'on peut faire est de fournir, ou d'aider à fournir, toutes les infrastructures nécessaires: routes, chemins de fer, et les autres installations de l'industrie moderne qu'il est du ressort du gouvernement de donner. ${ }^{106}$

Cette identification de l'intérêt collectif aux intérêts du capital fut typique de l'approche retenue à l'endroit de l'exploitation du domaine minier. Elle reflétait cependant une méconnaissance profonde des conséquences des mutations structurelles de l'industrie au $\mathrm{XX}^{\mathrm{e}}$ siècle, de la nature de l'investissement direct et de la corporation multinationale qui en fut le véhicule.

Toute politique minière doit ultimement être jugée par l'ampleur des retombées qui découlent de l'aliénation et de l'exploitation des ressources. En rétrospective, l'approche libérale sous-jacente à la politique québécoise fut erronée, et devant les échecs que constituaient, sur ce plan, l'expansion de l'industrie de l'amiante et du minerai de fer, les autorités ne crurent pas utile de la modifier ${ }^{107}$. La faiblesse principale de la politique minière est peut-être qu'elle ne fut jamais intégrée à une politique industrielle nationale ${ }^{108}$.

\section{Conclusion}

La croissance de l'industrie minière au Québec se réalisa essentiellement dans le contexte des importantes transformations économiques et technologiques du début du $\mathrm{XX}^{\mathrm{e}}$ siècle. Pour des raisons de proximité géographique et de sécurité d'approvisionnements, l'industrie minière québécoise devint l'un des nouveaux secteurs miniers mondiaux dont les matières premières furent en grande partie réservées au profit des principaux centres manufacturiers existants. La structure de

\footnotetext{
106 Roberts, op. cit., 67. La déclaration du ministre référait implicitement au projet de la Noranda Mines; l'honorable Perreault devint l'un des directeurs de l'entreprise en mars 1937 et le demeura jusqu'en 1948, ibid., 214.

107 Devant des échecs analogues à ceux du Québec, les gouvernements de plusieurs pays producteurs et exportateurs de matières premières vinrent à jouer un rôle plus actif dans l'exploitation, en vue de hausser leurs apports économiques; le Mexique et le Vénézuela, par exemple, créèrent des réserves nationales de minerais, et abaissèrent le degré de propriété étrangère de leurs industries minières tout en augmentant la part que l'État en retirait. Voir H. Gomez, «Venezuela's Iron Ore Industry», et M.S. Wionczek, «Foreign-owned Export Oriented Enclaves in a Rapidly Industrializing Economy: Sulphur Mining In Mexico», dans F. Mikesell, Foreign Investment in the Petroleum and Mineral Industries (Baltimore, 1971).

108 La révolution tranquille, avec l'organisation de Sidbec, et la période postérieure à 1975, avec la formation de la Société nationale de l'amiante, constituent des modifications importantes au laisser-faire économique traditionnel au Québec; une évaluation de ces deux «expériences» se situe cependant hors du cadre de cet article.
} 
développement du secteur fut satellitique, celui-ci étant complémentaire à l'industrialisation qui se déroulait à l'extérieur des frontières québécoises. Il y eut des dangers inhérents à une telle structure dépendante de développement; d'une part, la majeure partie des effets d'entraînement pouvait être réalisée ailleurs, et, d'autre part, les nouvelles économies régionales qui étaient créées au Québec risquaient d'être d'une grande fragilité tant à cause de leur spécialisation étroite que de la plus grande instabilité des marchés de minerais. La crise économique des dernières années illustre de façon spectaculaire la nature des pièges qui furent tendus dans ces régions; ce sont les propriétaires des ressources, État, travailleurs et travailleuses qui, aujourd'hui, en paient la note, les actionnaires privés s'en tirant beaucoup mieux.

La politique du développement minier au Québec contribua peu à faire bénéficier la population des immenses richesses qui furent appropriées en son nom en 1880 lors de l'adoption de l'Acte général des mines de Québec. Au XX $\mathrm{X}^{\mathrm{e}}$ siècle, la politique minière favorisa même la réservation du domaine minier au profit d'entreprises qui étaient intégrées à des opérations manufacturières étrangères. La politique minière québécoise devint en quelque sorte une police d'assurance aux industries manufacturières des États-Unis et de l'Ontario. Elle aurait pu être plus sélective. L'expérience historique et la conjoncture actuelle suggèrent qu'il pourrait être utile de réévaluer la politique minière afin que le secteur s'intègre mieux à l'économie du Québec et génère des retombées économiques plus importantes pour la collectivité. 